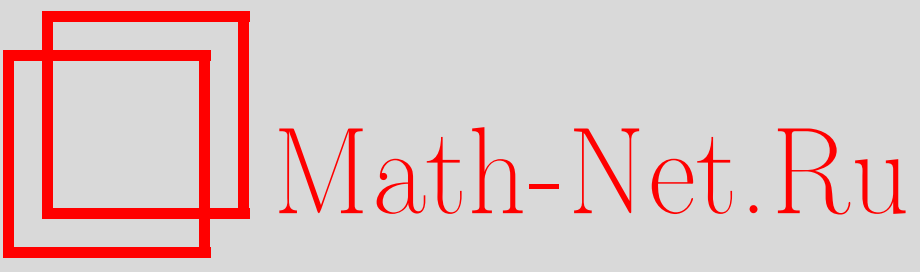

Н. А. Тюрин, Инстантоны и монополи, УМН, 2002, том 57, выпуск 2, 85-138

DOI: https://doi.org/10.4213/rm497

Использование Общероссийского математического портала Math-Net.Ru подразумевает, что вы прочитали и согласны с пользовательским соглашением

http://www.mathnet.ru/rus/agreement

Параметры загрузки:

IP: 54.198 .67 .100

26 апреля 2023 г., 13:29:18 
УДК 514.7

\title{
ИНСТАНТОНЫ И МОНОПОЛИ
}

\author{
Н. А. Тюрин
}

В настоящем обзоре представлены основные понятия и конструкции калибровочных теорий, а именно, теории Дональдсона, теории Зайберга-Виттена и связьвающей их теории В-монополей. В рамках дифференциальной геометрии эти теории доставляют новые инварианты гладких структур в размерности 4. Введение таких новых калибровочных инвариантов позволило решить многие проблемы в современной геометрии. Техника, разработанная в рамках этих теорий, доставляет новые современные методы исследований как в гладкой геометрии, так и в прикладных вопросах математической физики. Не претендуя на детальную полноту, данный обзор нацелен на то, чтобы представить предмет в максимальной широте и доступности.

Библиография: 27 названий

\section{СОДЕРЖАНИЕ}

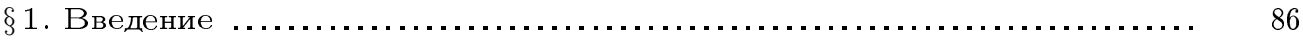

$\S 2$. Классическая теория Дональдсона ............................. 89

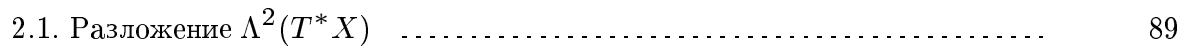

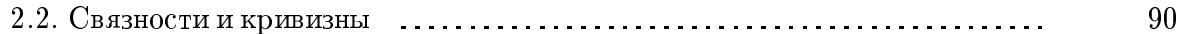

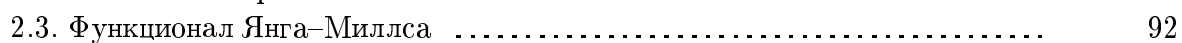

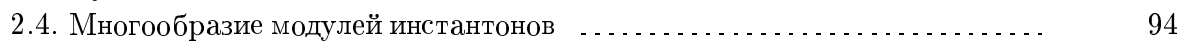

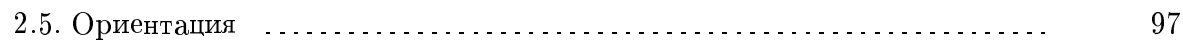

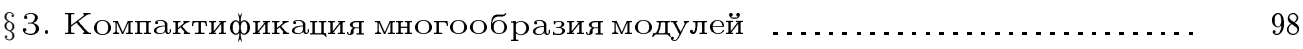

3.1. Идеальные связности ........................................ 98

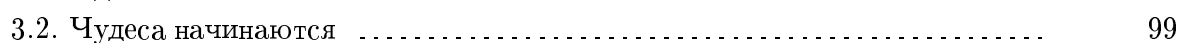

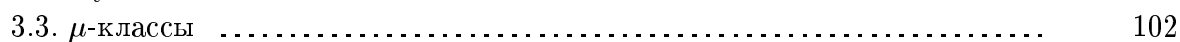

3.4. Инварианты Дональдсона . . . . . . . . . . . . . . . . . . . . . . . . . . . . . . . 104

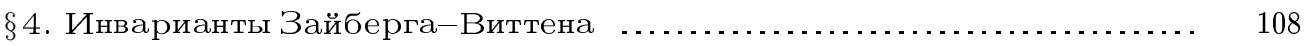

4.1. Алгебры Клифффорда .......................................... 108

4.2. Спинорные расслоения …................................ 111

4.3. Уравнение А-монополя ... . . . . . . . . . . . . . . . . . . . . . . . . . . . . . . . . 114

4.4. Инварианты Зайберга-Виттена .............................. 118

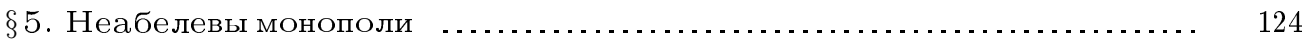

5.1. Конфигурационное пространство ... . . . . . . . . . . . . . . . . . . . . . . . . . 124

5.2. Инфинитезималшная теория деформации монополя вблизи инстантона . 129

5.3. Построение формалњного решения ............................. 131

5.4. Особенности многообразия модулей В-монополей ... . . . . . . . . . . . . 134

$\S 6$. Дополнение: гладкости и узлы ................................ 135

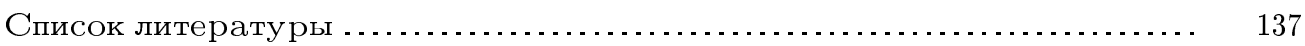

(C) Н.А. Тюрин 2002 


\section{$\S 1$. Введение}

П. А.М. Дирак в предисловии к первому изданию книги "Принципы квантовой механики" [3] определил понятие физической величины, наблюдаемой нами, как некоторый инвариант набора преобразований. Законы поведения в некоторой системе суть законы, перестановочные со всеми преобразованиями этой системы. Обшая цель физики представляется тогда как нахождение уравнений, инвариантных относительно как можно больших групп преобразований.

Такой подход близок и понятен и сердцу, и уму любого математика: в каждом разделе математики найдутся методы, связанные с нахождением и изучением инвариантов по действию соответствуюших групп преобразований. Инварианты заменяют нам объекты в наших рассмотрениях, и вопросы классификации объектов разрешаются в терминах численных характеристик, остаюшихся неизменньми под действием групп преобразований. Такие вопросы классификации, как и вопросы координатизации, указывают основной путь развития предмета.

Однако сразу встает вопрос - а какие это групшы и на каких многообразиях они действуют? Не успели математики разобраться с классической теорией инвариантов действия конечномерных групп Ли, как интересы геометрии потребовали перехода к рассмотрению бесконечномерных калибровочных групп. При таком переходе далеко не все средства остаются применимыми (поэтому такой переход и обобщением-то назвать трудно), тем не менее необходимо сох ранить фундаментальные методы, среди которых одним из наиболее полезных является, например, симплектическая редукция. Такая динамика использования понятий не свойственна излишне стабильной математической практике. Очень часто у специалистов в той или иной области вызьвает крайнее удивление, как их "собственньй”, “законньй метод” превосходно срабатьвает во вполне далекой от этого предмета задаче, иногда в замаскированном виде.

Не так давно С. Дональдсоном [6] были предложены к рассмотрению совсем уж экзотические геометрические объекты - многообразия модулей инстантонов на четырехмерных многообразиях. Их можно использовать как пленки кобордизмов в дифференциальной топологии. В частности, такие многообразия модулей могут быть использованы для определения инвариантов подлежашего гладкого четырехмерного многообразия $X$. Они определяются фундаментальным классом $[\mathscr{M}]$ многообразия модулей инстантонов и классами когомологий на пространстве классов калибровочно эквивалентных неприводимых связностей $A^{*} / \mathscr{G}$.

Модули инстантонов доставляют инварианты подлежащего многообразия в форме статсумм и корреляторов, а это то, чем интересуются физики. Поэтому вполне предсказуема связь между квантовой теорией поля и дифференциальной геометрией, возникаюшая при изучении инстантонов. Простейший пример: Э. Виттен в [27] показал, что можно определить, по крайней мере формально, квантовую теорию поля, для которой статсумма вида

$$
W(t)=\iint e^{-t S(A, \phi)} \mathscr{D} A \mathscr{D} \phi
$$

не зависит от $t$ и доставляет дифференщиально-топологические инварианты подлежащего многообразия $X$. Здесь интеграл берется по пространству пар $(A, \phi)$, где $A-$ связность, а $\phi$ - некоторое вспомогательное поле (поле Хиггса). Производя интегрирование сначала по $\phi$, возможно представить $W(t)$ как интеграл по пространству 
связностей. При стремлении параметра $t$ к нулю подьнтегральное выражение локализуется вблизи инстантонов, и Виттен предположил, что мы снова получаем те же инварианты, т.е. полиномы Дональдсона.

Чуть позднее Виттен совместно с Н. Зайбергом при исследовании механизма трех фаз квантового вакуума в суперсимметричных теориях Янга-Миллса (конденсации, конфайнмента и фазы Хиггса) использовали неприводимые $(\phi \neq 0)$ классические минимумы функционала $S$ (см. [7], [18]). Редуцируя связности к бесследовьм связностям Леви-Чивита для спинорных расслоений, они построили новые инварианты четырехмерных многообразий, тесно связанные с инвариантами Дональдсона, но зачастую гораздо более простые в вычислениях. Инварианты Зайберга-Виттена четырехмерного многообразия $X$ определяются количеством решений системы дифференциальных уравнений для экстремалей функционала $S$ при выборе $\operatorname{Spin}^{\mathbb{C}}$-структуры на $X$. То есть строится отображение из классов эквивалентности $\operatorname{Spin}^{\mathbb{C}}$-структур (возможных классов Черна детерминанта спинорного расслоения при отсутствии 2-кручения во вторых когомологиях подлежашего многообразия) в целые числа.

Введение этой системы, получившей название уравнения А-монополя, сразу привело к прорьву плотины нерешенных задач. Приведем два примера, ярко иллюстрирующих степень полезности и применимости уравнения А-монополя в алгебраической и симплектической геометрии.

П. Кронхеймер и Т. Мровка в [13] показали, как использование уравнения монополя легко (полное доказательство - на трех страницах, случай небьвальй в современной практике) приводит к доказательству гипотезы Тома - "род алгебраической кривой в $\mathbb{C P}^{2}$ равен нижней границе рода любых 2-многообразий, представляюших один и тот же класс гомологий”. (Тот же минимум реализуется и для псевдоголоморфных кривых.)

Род гладкой алгебраической плоской кривой степени $d$ равен $g=(d-1)(d-2) / 2$. Отсюда не слишком неожиданной вьглядит

ТеОРема 1.1. Пусть $\Sigma$ - ориентированное двумерное многообразие, гладко вложенное в $\mathbb{C P}^{2}$ так, что представляет тот же класс гомологий, что и некоторая алгебрачческая кривая степени $d$. Тогда род $\Sigma$ больше или равен $(d-1)(d-2) / 2$.

Очевидно, что, приклеивая к римановой поверхности очень маленькие ручки, мы не изменим класс когомологий поверхности, но как угодно увеличим ее род. Поэтому вопрос именно в том, каков минимальньй род, реализуемьй кривыми.

Другой интереснейший результат представлен К. Таубсом в [19], [20]: на гладком ориентированном компактном многообразии $X$ симплектическая структура $\omega$, совместимая с ориентацией, задает стягиваемьй конус почти комплексных структур, эрмитово совместимых с ней. Выбрав одну, мы немедленно получаем метрику $g$ на $X$ и класс Черна $c_{1}$ комплексного касательного расслоения на $X$. Этот класс - аналог канонического класса келеровой поверхности и может быть взят в качестве $\mathrm{Spin}^{\mathbb{C}}$-структуры на $X$. Будучи целочисленным, этот класс не зависит от выбора почти комплексной структуры, а зависит только от деформационного класса симплектический структуры. Главная теорема Таубса утверждает, что в этом случае инвариант Зайберга-Виттена для класса $c_{1}$ и метрики $g$ равен \pm 1 .

Вообще необходимо заметить, что в симплектическом (как и в более специальном алгебро-геометрическом) случае уравнение монополя имеет более простой вид. При- 
чина в том, что в этом случае спинорное расслоение раскладьвается в прямую сумму линейных расслоений, одно из которых - каноническое (см. §3).

Классы Зайберга-Виттена являются аналогами канонических классов алгебраических поверхностей, а вся (конечная) совокупность их инвариантна относительно действия групшы диффеоморфизмов (если $b_{2}^{+}>1$ ) подлежашей гладкой структуры.

Краткость доказательства, предложенного Кронхеймером и Мровкой, на самом деле весьма обманчива. Чтобы полностью привести это доказательство, необходимо потребовать от читателя информированности в двух традиционно различных (несмотря на усиленную пропаганду их объединения) областях математики - PDE и дифференциальной топологии. Цель этой статьи и заключается в том, чтобы объединить эти два фронта идей для широкого круга лиц, заинтересованных в том, чтобы замечательные результаты - подобные гипотезе Тома - доказывались в три страницы и открывали возможность дальнейших исследований.

Сила результатов теории Дональдсона спровоцировала желание эту теорию “упростить". Это приводит к терминологическим недоразумениям. Например, частный случай монополей, используемых в дифференциальной геометрии, называются абелевыми монополями. Это недоразумение. Связности в спинорных монополях, конечно, неабелевы. Только бесследовая часть этих связностей фиксирована и равна бесследовой части связности Леви-Чивита, так что свободной переменной остается только диагональная часть, т.е. абелева связность.

Конечно, при исходном физическом подходе подобные недоразумения исключены в силу самого физического смысла уравнений. Поэтому в процессе нашего описания теории мы обязаны обращаться к физическим истокам и в историческом и в идейном аспектах. А широко используемые ньне в дифференциальной топологии монополи будем назьвать А-монополями.

С другой стороны, неабелевы монополи позволяют наглядно, геометрически соединить инварианты Дональдсона с инвариантами Зайберга-Виттена. Эта конструкция (см. [16]) абсолютно аналогична конструкции пленки кобордизма между 4-многообразием с определенной формой пересечения и несвязным объединением комплексных проективных поверхностей (см. ниже), которой Дональдсон открыл новейший этап четырехмерной дифференциальной топологии.

Вложение многообразия модулей инстантонов в многообразие модулей монополей можно использовать также и для доказательства сушествования решений наших сложных PDE (абсолютно параллельно тому, как это мы делаем для bubbling-инстантонов). Дополнительная сложность - задача описания, как себя ведут решения уравнения неабелева монополя при приближении к инстантонам. Пределами решений уравнения монополя при стремлении спинорной части к нулю могут быть только инстантоны подскока, т.е. такие asd-связности, которые допускают существование гармонического спинора, и каждый инстантон подскока реализуется как предел монополей (см. $\S 4)$. Заметим, что последнее утверждение отвечает еще и на вопрос о существовании самих В-монополей.

Для алгебраического геометра эти конструкции вполне привычны: подмногообразие инстантонов подскока в многообразии модулей инстантонов - прямой аналог первого члена фильтрации Брилля-Нётера якобиана кривой (см. [15]).

Замечательно, что, как и в классической теории Брилля-Нётера, в гладкой части многообразия модулей классы когомологий локусов Брилля-Нётера представляются 
классами Черна индексного расслоения оператора Дирака.

Геометрическое представление этих классов Черна продолжается и на особенности многообразия модулей. Сравнение классов этих продолжений и классов индексного расслоения эквивалентно задаче выражения классов Зайберга-Виттена через классы Дональдсона.

В тексте мы приводим все определения и конструкции калибровочной дифференциальной геометрии, поэтому многие ссылки на литературу преднамеренно опущены: мы вынуждены пренебречь многообразием литературы по данным темам (особенно по инстантонам), поскольку это затруднило бы для читателя первое ознакомление с этими новыми предметами, а, с другой стороны, наличие недоразумений, аналогичных упоминаемой абелевости монополей Зайберга-Виттена, вьнудило бы нас параллельно процитировать и все физические тексты по этой теме. При этом мы сразу даем ссылку на основные источники для каждого параграффа, чтобы не испещрять текст в конце каждого абзаца или после каждой формулы. Выход один - сделать текст как можно более самозамкнутым. Для материала $\S \S 2$ и 3 (инварианты Дональдсона) необходимы источники [2] и [6], а для материала $\S 4$ (инварианты Зайберга-Виттена) [13] и [26]. С §5 дело обстоит проще: как справочный материал достаточно использовать [16] и [24].

Наконец в $\S 6$ (Добавлении) мы от калибровочных теорий возвращаемся к классической топологии, следуя (насколько это возможно) красивым конструкциям Р. Финташела и Р. Стерна [9].

В заключение введения пользуюсь случаем выразить глубокую благодарность Сергею Петровичу Новикову и Виктору Матвеевичу Бухштаберу за помощь в подготовке и публикации настоящего обзора.

\section{§. Классическая теория Дональдсона}

2.1. Разложение $\Lambda^{2}\left(T^{*} X\right)$. Как обычно в теории Дональдсона, мы будем рассматривать $(X, g)$ - четырехмерное односвязное компактное ориентируемое риманово многообразие. Центральное место в теории занимает разложение пространства 2-форм по действию оператора Ходжа *.

Оператор определяется как композиция естественной метрики на формах и внешнего произведения:

$$
\alpha \wedge * \beta=(\alpha, \beta) d \mu
$$

где $d \mu$ - элемент объема. На четырехмерном многообразии $*$ отображает 2-формы в 2-формы, и очевидным образом $* *=\mathrm{id}_{\Lambda^{2}}$. Пространства автодуальных и антиавтодуальных форм (обозначаемые как $\Omega^{+}{ }_{\text {и }} \Omega^{-}$соответственно), определяемые как собственные подпространства с собственными значениями \pm 1 оператора $*$, являются сечениями расслоений $\Lambda^{+}$и $\Lambda^{-}$:

$$
\Lambda^{2}=\Lambda^{+} \oplus \Lambda^{-}, \quad \alpha \wedge \alpha= \pm|\alpha|^{2} d \mu \rightarrow \alpha \in \Gamma\left(\Lambda^{ \pm}\right) .
$$

На 4-многообразии такое разложение зависит только от конформного класса метрики. 
На пространствах сечений $\Omega^{p}$ имеется кроме обычного дифференциала $d$ формально сопряженный $d^{*}$, определяемьй как:

$$
d^{*}: \Omega^{p} \rightarrow \Omega^{p-1}, \quad \int(d \alpha, \beta) d \mu=\int\left(\alpha, d^{*} \beta\right) d \mu,
$$

т.е. в ориентируемом случае $d^{*}= \pm * d *$.

Теорема Ходжа утверждает, что каждый класс действительных когомологий представляется единственным образом гармонической формой $\alpha$ такой, что

$$
d \alpha=d^{*} \alpha=0 \text {. }
$$

Гармоничность формы сохраняется под действием оператора *, поэтому задание метрики определяет разложение

$$
H^{2}(X, \mathbb{R})=H^{+} \oplus H^{-}
$$

на автодуальные и антиавтодуальные гармонические формы. Это следует из того, что они являются максимальными положительным и отрицательным подпространствами для формы пересечения, рассматриваемой как внешнее произведение на классах де Рама. Поэтому

$$
\operatorname{dim} H^{+}=b^{+}, \quad \operatorname{dim} H^{-}=b^{-},
$$

где $\left(b^{+}, b_{-}\right)$- сигнатура формы пересечения на $X$. То же можно получить и из построения половины комплекса де Рама. А именно, определим оператор $d^{+}$как композицию, действуюшую из пространства 1-форм в $\Omega^{+}$, обычного внешнего дифференцирования и алгебраической проекции $(1 / 2)(1+*)$. Получаем комплекс

$$
\Omega^{0} \stackrel{d}{\longrightarrow} \Omega^{1} \stackrel{d^{+}}{\longrightarrow} \Omega^{+},
$$

которьй есть, грубо говоря, половинка обычного комплекса. Если $X$ компактно, тогда когомологии этого комплекса естественно отождествляются с $H^{0}(X, \mathbb{R}), H^{1}(X, \mathbb{R})$ и $H^{+}$.

2.2. Связности и кривизны. Напомним, что главным $G$-расслоением $P$ над гладким многообразием $X$ называется многообразие с заданным гладким правым действием группы $G$ таким, что пространство орбит есть подлежашее $X$.

Можно предложить три определения связности на главных расслоениях, а именно:

а) как поле горизонтальных подпространств $H \subset T P$, трансверсальных к слою проекции $\pi: P \rightarrow X$;

б) как 1-форму $A$ на $P$ со значениями в алгебре Ли групшы, инвариантную по действию $G$, ограничение которой на каждьй слой совпадает с канонической правоинвариантной формой;

в) для любого представления $G$ мы получаем векторное расслоение $E$ над подлежашим многообразием, и связность может быть определена через ковариантную производную на $E$, т.е. линейное отображение

$$
\nabla: \Omega^{0}(E) \rightarrow \Omega^{1}(E),
$$

где $\Omega^{p}(E)$ - пространство векторнозначных $p$-форм, или сечения расслоения $\Lambda^{p} T^{*} X \otimes E$; отображение $\nabla$ должно удовлетворять правилу Лейбнища

$$
\nabla(f \cdot s)=f \cdot \nabla s+d f \cdot s
$$

для любого сечения расслоения $E$ и функции $f$. 
Кроме того, если на $E$ заданы дополнительные структуры, связность должна быть с ними согласована. Например, при задании метрики связность должна удовлетворять условию:

$$
d(s, t)=(\nabla s, t)+(s, \nabla t)
$$

(введение дополнительных структур соответствует тому, что группа становится меньше (редуцируется к подгруппе), например, эрмитова метрика предполагает, что группа не полная линейная, а унитарная).

Все три определения связности суть одно и то же.

Обычно используется третье определение, но всегда полезно видеть за ним и все три. Тогда, например, очевидно, что связность на расслоении $E$ есть одновременно и связность на $\mathrm{ad} E$. Действительно, связность на векторном расслоении есть и связность на главном расслоении с группой Aut $E$ и той же базой. Но мы можем рассмотреть два разных представления этого главного расслоения - стандартное и присоединенное. В первом случае мы снова получим $E$, во втором ad $E$, оба векторных расслоения снабжены связностью, которая есть одна и та же связность.

Из обших свойств связностей хотелось бы указать следуюшие:

а) на тривиальном расслоении $\mathbb{C}^{n}=\mathbb{C}^{n} \times X$ есть выделенная связность - стандартная, ковариантное дифференцирование, задаваемое ею, есть просто дифференцирование векторнозначных функций;

б) связность - объект ковариантньй;

в) связность определяет параллельньй перенос слоев вдоль пути на подлежащем многообразии;

г) разность двух связностей есть 1-форма; пусть $a$ - некоторая связность на $E$, а $\omega$ - элемент пространства $\Omega^{1}(\mathrm{ad} E)$; тогда определена новая эрмитова связность $a+\omega$, причем $\omega$ действует алгебраически на сечениях расслоения посредством очевидного спаривания

$$
\Omega^{0}(E) \times \Omega^{1}(\text { End } E) \rightarrow \Omega^{1}(E),
$$

а ограничение на ad $E \subset$ End $E$ соответствует тому, что новая связность согласована со структурной группой $E$.

Таким образом, пространство эрмитовых связностей $\mathscr{A}$ есть аффинное пространство над векторным $\Omega^{1}(\mathrm{ad} E)$.

Введем внешнюю производную на пространствах $\Omega^{p}(E)$,

$$
d_{a}: \Omega^{p}(E) \rightarrow \Omega^{p+1}(E)
$$

отображающую $\Omega^{p}(E)$ в $\Omega^{p+1}(E)$ по правилам

$$
\begin{gathered}
d_{a}=\nabla_{a} \text { на } \Omega^{0}(E), \\
d_{a}(\omega \wedge \eta)=(d \omega) \wedge \eta+(-1)^{p} \omega \wedge d_{a} \eta,
\end{gathered}
$$

если $\omega \in \Omega^{p}$ и $\eta \in \Omega^{q}(E)$. Заметим сразу, что такое же внешнее диффференцирование аналогичным образом вводится и на пространствах $\Omega^{p}(\operatorname{ad} E)$, причем эти два оператора действуют согласованно. 
Оператор $d_{a}$ очень похож на обьчное внешнее дифференцирование, но комплекса, подобного комплексу де Рама, с его помощью определить не удастся. Препятствием к этому является кривизна связности $a$.

Кривизна есть последовательное применение оператора $d_{a}$ дважды, а именно, $F_{a}=$ $d_{a} d_{a}$. Проверкой правила Лейбница устанавливается, что кривизна есть оператор алгебраический, $F_{a} \in \Omega^{2}(\operatorname{ad} E)$.

Нетрудно вьвести формулу для кривизны возмушенной связности:

$$
F_{a+\omega}=F_{a}+d_{a}(\omega)+\omega \wedge \omega
$$

где последнее выражение обозначает композицию внешнего произведения и естественного умножения в ad $E \subset$ End $E$.

Тождество Бианки утверждает для любой связности равенство

$$
d_{a}\left(F_{a}\right)=0
$$

Пользуясь метрикой, можно ввести оператор, формально сопряженный оператору внешнего дифференцирования:

$$
d_{a}^{*}: \Omega^{p}(E) \rightarrow \Omega^{p-1}(E)
$$

определяемый равенством

$$
\int_{X}\left(d_{a} \phi, \psi\right) d \mu=\int_{X}\left(\phi, d_{a}^{*} \psi\right) d \mu
$$

для любых форм подходящего порядка.

Разложение (2.2) очевидным образом продолжается и на пространство

$$
\Omega^{2}(\operatorname{ad} E)=\Omega^{+}(\operatorname{ad} E) \oplus \Omega^{-}(\operatorname{ad} E)
$$

поэтому мы имеем

$$
F_{a}=F_{a}^{+}+F_{a}^{-}, \quad F_{a}^{ \pm} \in \Omega^{ \pm}(\operatorname{ad} E) .
$$

Связность $a$ называется автодуальной, если $F_{a}^{-}=0$, и антиавтодуальной, если $F_{a}^{+}=0$.

Напомним еще раз, что разложение (2.2) конформно инвариантно, значит, и условия автодуальности и антиавтодуальности обладают таким же свойством.

2.3. Функционал Янга-Миллса. Напомним, что согласно теории Черна-Вейля для любого комплексного расслоения $E$ и произвольной связности $a$ на его сечениях первый класс Черна $c_{1}(E)$ представляется замкнутой формой $\frac{i}{2} \pi \operatorname{Tr}\left(F_{a}\right)$. След квадрата кривизны при этом выражается следуюшим образом:

$$
\left[\frac{1}{4 \pi^{2}} \operatorname{Tr}\left(F_{a}^{2}\right)\right]=2 c_{2}(E)-c_{1}^{2}(E) \in H^{4}(X) .
$$


Для четырехмерных многообразий - односвязных, ориентируемых, компактных которые обычно и рассматриваются в теории Дональдсона, и для $\mathrm{SU}(2)$-связностей имеем в качестве следствия предыдущей формулы

$$
c_{2}(E)=\frac{1}{8 \pi^{2}} \int_{X} \operatorname{Tr}\left(F_{a}^{2}\right) \in \mathbb{Z}
$$

Разберем условие антиавтодуальности. Заметим, что в алгебре кососимметрических матриц $\operatorname{Tr}\left(\omega^{2}\right)=-|\omega|^{2}$. Вместе с определением разложения 2-форм это дает

$$
\operatorname{Tr}\left(F_{a}^{2}\right)=-\left(\left|F_{a}^{+}\right|^{2}+\left|F_{a}^{-}\right|^{2}\right) d \mu,
$$

а значит, после интегрирования по компактной базе получаем

$$
8 \pi^{2} c_{2}(E)=\int_{X}\left|F_{a}^{-}\right|^{2} d \mu-\int_{X}\left|F_{a}^{+}\right|^{2} d \mu .
$$

Таким образом, абсолютное значение $c_{2}$ задает нижнюю границу значений функционала Янга-Миллса, вид которого очень прост - квадрат $L^{2}$-нормы кривизны равен

$$
\left\|F_{a}\right\|^{2}=\int_{X}\left|F_{a}\right|^{2} d \mu=\int_{X}\left|F_{a}^{-}\right|^{2} d \mu+\int_{X}\left|F_{a}^{+}\right|^{2} d \mu .
$$

Если $c_{2}$ положителен, то очевидным образом критерием антиавтодуальности будет условие $\left\|F_{a}\right\|^{2}=8 \pi^{2} c_{2}(E)$.

Уравнение Эйлера-Лагранжа для функционала $\left\|F_{a}\right\|^{2}$ на пространстве связностей, называемое уравнением Янга-Миллса, имеет вид

$$
d_{a}^{*} F_{a}=0 .
$$

Эта удивительная простота теории Янга-Миллса невольно наводит на мысль об универсальной применимости ее во многих областях. Но еще более поразителен тот факт, что в четырехмерном случае функционал конформно инвариантен. Действительно, в случае размерности $d$ умножение метрики на $c$ дает в 2-формах множитель $c^{-2}$, в то же время элемент объема изменяется в $c^{d}$ раз. Поэтому интеграл $\int_{X}\left|F_{a}\right|^{2} d \mu$ преобразуется к виду $\int_{X} c^{4-d}\left|F_{a}\right|^{2} d \mu$ и остается неизменньг, в точности когда $d=4$.

Обратим внимание на то, что нижняя граница значения функционала задается в случае SU(2)-связности только вторым классом Черна. Это следствие того, что первьй класс Черна $\mathrm{SU}(2)$-расслоения равен нулю.

Одновременно для унитарных и ортогональных связностей введем характеристическое число $k(E)$, которое:

a) для $\mathrm{SU}(r)$-расслоений равно, как и вьше, $k(E)=c_{2}(E)$;

б) для $\mathrm{U}(r)$-расслоений равно $k(E)=c_{2}(E)-\frac{1}{2} c_{1}^{2}(E)$;

в) для $\mathrm{SO}(r)$-расслоений (вешественных) равно $k(V)=-\frac{1}{4} p_{1}(V)$.

Напомним, что первый класс Понтрягина $p_{1}(V)$ определяется формулой $p_{1}(V)=$ $c_{2}(V \otimes \mathbb{C}) \in H^{4}(X, \mathbb{Z})$.

После всего сказанного становится очевидным, что если расслоение $E$ над $X$ допускает антиавтодуальные связности, то $k(E) \geqslant 0$, а если $k(E)=0$, то все антиавтодуальные связности плоские. 
2.4. Многообразие модулей инстантонов. Построение многообразия модулей инстантонов разбивается на два шага: первый заключается в решении уравнения антиавтодуальности (которое мы будем для краткости так и назьвать - уравнение инстантона), второй - в разбиении пространства решений (бесконечномерного) на орбиты по действию группы калибровочных преобразований, факторизации по действию групш, в результате чего получается конечномерноегладкое для обшей метрики многообразие, которое возможно компактифицировать.

Факторизация по действию групш необходима - с бесконечномерным пространством решений трудно связать какие-либо объекты, имеющие математический смысл. Заметим, однако, что физики для получения статсумм вида (1.1) смело интегрируют по всем связностям: метод перевала приводит к рассмотрению только малых окрестностей экстремалей функционала Янга-Миллса, в частности - абсолютных минимумов, т.е. инстантонов.

Для построения классов эквивалентности на пространстве связностей необходимо (и обычно применяется) пополнение по соболевским нормам. Для этого фиксируется некоторое $l \geqslant 2$ и сечения расслоения пополняются по норме $L_{l}^{2}$. Тогда связности наделяются $L_{l-1}^{2}$-нормой, а кривизны $-L_{l-2}^{2}$-нормой.

Группа калибровочных преобразований $\mathscr{G}$ (или, как мы будем иногда, допуская математическую неряшливость языка, говорить - калибровочная групша) есть просто группа $L_{l}^{2}$-автоморфизмов расслоения $E$.

Определим факторпространство

$$
\mathscr{B}=\mathscr{A} / \mathscr{G}
$$

Очевидно, что метрика на пространстве связностей, определяемая как

$$
\|a-b\|=\left(\int_{X}|a-b|^{2} d \mu\right)^{1 / 2}
$$

(тождественная метрике на пространстве 1-форм со значениями в $\operatorname{ad} E$ ), сохраняется при действии калибровочной группы одновременно на обе связности, поэтому для более точного отражения топологической структуры на $\mathscr{B}$ необходимо ввести расстояние между классами связностей.

Если $[a]$ и $[b]$ - орбиты связностей, тогда определим расстояние как

$$
\tau([a],[b])=\inf \|a-u(b)\|, \quad u \in \mathscr{G} .
$$

И, естественно, это индуцирует $\tau$ - метрику на факторпространстве $\mathscr{B}$.

Для описания окрестности класса $[a]$ в факторпространстве $\mathscr{B}$ воспользуемся следующей конструкцией. Пусть

$$
T_{a, \varepsilon}=\left\{\omega \in \Omega^{1}(\operatorname{ad} E) \mid d_{a}^{*}(\omega)=0,\|\omega\|<\varepsilon\right\}
$$

где, договоримся, под нормами будем понимать соболевские нормы тех индексов, которые мы присвоили пространствам в начале п. 2.4. Тогда окрестность орбиты, представляемой связностью $a$, может быть описана как некоторая факторизация $T_{a, \varepsilon}$ при достаточно малом $\varepsilon$. 
Заметим, что условие $d_{a}^{*}(\omega)=0$ в определении (2.29) очень просто истолковьвается. Действительно, если форма $\omega$ точна, т.е. представляется в виде $d_{a} \lambda$, где $\lambda$-просто функция со значениями в эндоморфизмах, то нетрудно видеть, что $\tau([a+\omega],[a])=0$.

Условие $d_{a}^{*}(\omega)=0$ означает, что связности $a+\omega$ и $a$ находятся в кулоновской калибровке.

Чтобы объяснить, как факторизуется $T_{a, \varepsilon}$, введем для каждой связности подгруппу калибровочных преобразований

$$
\Gamma_{a}=\{u \in \mathscr{G} \mid u(a)=a\}
$$

Как замкнутая подгруппа калибровочной групшы $\Gamma_{a}$ является групой Ли. Ее алгебра Ли определяется просто как ядро $\operatorname{ker} d_{a} \subset \Omega^{0}(\operatorname{ad} E)$. Очевидно, группа $\Gamma_{a}$ действует на $\Omega^{1}(\operatorname{ad} E)$ и, следовательно, на $T_{a, \varepsilon}$.

Оказьвается, что для достаточно малых $\varepsilon$ проекция $\pi: \mathscr{A} \rightarrow \mathscr{B}$ индуцирует гомеоморфизм между фактором $T_{a, \varepsilon} / \Gamma_{a}$ и окрестностью орбиты $[a]$.

Групш $\Gamma_{a}$ классифицируют связности в следуюшем смысле. Если такая подгруппа минимальна - а это значит, что она в каждой точке совпадает с централизатором структурной групш расслоения, то мы будем называть соответствующую связность неприводимой.

Открытое подмножество неприводимых связностей мы будем обозначать $\mathscr{A}^{*} \subset \mathscr{A}$. Тогда фактор неприводимых связностей по действию калибровочной групшы $\mathscr{B}^{*} \subset \mathscr{B}$ локально изображается шарами $T_{a, \varepsilon}$ в гильбертовых пространствах $\operatorname{ker} d_{a}^{*} \subset L_{l-1}^{2}\left(\Omega^{1}(\operatorname{ad} E)\right)$. Это задает покрытие $\mathscr{B}^{*}$ картами, преврашаюшее это факторпространство в гладкое гильбертово многообразие неприводимых связностей.

Термин "приводимость" используется обычно в том смысле, что связность раскладьвается в прямую сумму пары связностей, действуюших на паре расслоений, прямая сумма которых составляет исходное расслоение. Это далеко не всегда равносильно тому, что изотропная подгруппа $\Gamma_{a}$ связности $a$ минимальна. Но в условиях наших рассмотрений (SU(2)-, U(2)-, $\mathrm{SO}(3)$-расслоение над односвязным многообразием) два эти условия совпадают.

В случае комплексного $\mathrm{SU}(2)$-расслоения приводимость соответствует разложению $E=L \oplus L^{-1}$, при этом ad $E=\mathbb{R} \oplus L^{\otimes 2}$. В случае вещественного - разложение имеет вид $\operatorname{ad} E=\mathbb{R} \oplus L$.

Как уже отмечалось, приводимые связности составляют особенности гильбертова многообразия $\mathscr{B}$. Самой особой является самая простая - тривиальная - связность. Состояшая из трех различных компонент, она представляется конусом над пространством, которое и само является особым.

Необходимо также добавить, что для $\mathrm{SU}(2)-$ или $\mathrm{SO}(3)$-связности калибровочно эквивалентные классы приводимых антиавтодуальных связностей в точности соответствуют парам $\pm c$, где $c$ - ненулевые классы в $H^{2}(X, \mathbb{Z})$, причем $c^{2}=-c_{2}(E)$ или $p_{1}(E)$ соответственно, но это верно, подчеркнем, только в односвязном случае.

Очевидно, что условие антиавтодуальности инвариантно при действии калибровочной группы. Это следует из инвариантности функционала Янга-Миллса, экстремалями которого являются эти связности. Поэтому на пространстве модулей $\mathscr{B}$ определяется подмножество $\mathscr{M}_{\text {asd }}$ - орбиты антиавтодуальных связностей по модулю действия калибровочной группы. A priori такие множества должны обозначаться как $\mathscr{M}_{\text {asd }}(l)$, в зависимости от индекса соболевских норм. Однако естественное вложение 
$\mathscr{M}_{\text {asd }}(l+1)$ в $\mathscr{M}_{\text {asd }}(l)$ оказывается гомеоморфизмом. Поэтому в дальнейшем индексы будут опускаться, за исключением тех случаев, когда это может привести к недоразумению.

$\mathscr{M}_{\text {asd }}$ называется многообразием модулей антиавтодуальных связностей или - короче - инстантонов.

Локально $\mathscr{M}_{\text {asd }}$ может быть описано так же, как и $\mathscr{B}$. Пусть $a-$ антиавтодуальная связность. Тогда определим отображение $\psi$ таким образом:

$$
\begin{gathered}
\psi: T_{a, \varepsilon} \rightarrow \Omega^{+}(\operatorname{ad} E), \\
\psi(\omega)=F^{+}(a+\omega)=d_{a}^{+}(\omega)+(\omega \wedge \omega)^{+} .
\end{gathered}
$$

Как и выше, фактор схемы нулей этого отображения по изотропной подгрупе $Z(\psi) / \Gamma_{a}$ гомеоморфен окрестности $[a]$ в $\mathscr{M}_{\text {asd }}$.

Заметим, что отображение $\psi$ - гладкое фредгольмово, дифференциал которого в нуле есть просто

$$
d_{a}^{+}: \operatorname{ker} d_{a}^{*} \rightarrow \Omega^{+}(\operatorname{ad} E) .
$$

Это следует из эллиптичности (и, значит, фредгольмовости) оператора $\delta_{a}=d_{a}^{*} \oplus d_{a}$, поскольку ограничение оператора в (2.32) совпадает с $\delta_{a}$. Размерность многообразия модулей инстантонов совпадает с индексом оператора $\delta_{a}$. Обозначим $s=\operatorname{ind} \delta_{a}$. Это число находится по теореме Атьи-Зингера и зависит только от топологических параметров подлежашего многообразия и расслоения $E$. А именно, для $\mathrm{SU}(2)$-расслоения получаем

$$
s=8 c_{2}(E)-3\left(1-b_{1}(X)+b_{2}^{+}(X)\right),
$$

а для $\mathrm{SO}(3)$-расслоения

$$
s=-2 p_{1}(E)-3\left(1-b_{1}(X)+b_{2}^{+}(X)\right) .
$$

В локальном описании уравнения $d_{a}^{*}(\omega)=0$ и $d_{a}^{+}(\omega)+(\omega \wedge \omega)^{+}=0$ играют разную роль. Второе есть собственно уравнение антиавтодуальности, первое же описывает локально срез (в кулоновской калибровке) на пространстве орбит в некоторой окрестности связности $a$. Такой срез может быть выбран и другими способами, поэтому правильнее представлять себе линеаризацию объекта следующим образом.

Рассмотрим деформационньй комплекс

$$
\Omega^{0}(\operatorname{ad} E) \stackrel{d_{a}}{\longrightarrow} \Omega^{1}(\operatorname{ad} E) \stackrel{d_{a}^{+}}{\longrightarrow} \Omega^{+}(\operatorname{ad} E) .
$$

Условие антиавтодуальности равносильно тому, что это действительно комплекс, определяющий группы когомологий $H_{a}^{0}, H_{a}^{1}, H_{a}^{2}$. Средние когомологии представляют линеаризацию уравнений инстантона по модулю действия калибровочной групшы

$$
H_{a}^{1}=\operatorname{ker} d_{a}^{+} / \operatorname{im} d_{a}
$$

и теория Ходжа доставляет нам естественное отождествление

$$
H_{a}^{1} \cong \operatorname{ker} \delta_{a}, \quad H_{a}^{2}=\operatorname{coker} d_{a}^{+} \cong \operatorname{ker} d_{a} \subset \Omega^{+}(\operatorname{ad} E)
$$


В терминах этого комплекса число $s$ есть просто эйлерова характеристика, взятая с обратным знаком:

$$
s=\operatorname{dim} H_{a}^{1}-\operatorname{dim} H_{a}^{0}+\operatorname{dim} H_{a}^{2} .
$$

Локальной моделью $\mathscr{M}_{\text {asd }}$ будет фактор $f^{-1}(0) / \Gamma_{a}$, где

$$
f: H_{a}^{1} \rightarrow H_{a}^{2}
$$

- эквивариантное относительно изотропной подгруппы отображение. Функция $f$ может быть выбрана вещественной аналитической, следовательно, многообразие модулей инстантонов - вещественно аналитическое. Обычно такая функция назьвается отображением Кураниши.

В случае неопределенной формы пересечения (см. ниже) и общей метрики многообразие модулей не содержит приводимых связностей; для неприводимых связностей по теореме, доказанной К. Уленбек, вторые когомологии деформационного комплекса (2.35) равны нулю. Заметим, что и для случая, когда обшие метрики допускают приводимые антиавтодуальные связности (т.е. когда форма пересечений 2-циклов определена), все равно $H_{a}^{2}=0$. Поэтому в любом случае (для общей метрики) многообразие модулей инстантонов гладкое или гладкое почти всюду, кроме конечного числа точек, линки которых гомеоморфны проективньм пространствам.

Наконец, чтобы использовать многообразия модулей как геометрические объекты (например, пленки бордизмов), необходимо еще одно важное свойство многообразия модулей инстантонов - ориентируемость.

2.5. Ориентация. Рассмотрим сначала случай $\mathrm{SU}(2)$-расслоения. Возьмем на многообразии модулей $\mathscr{M}_{\text {asd }}$ открытое подмножество $\mathscr{M}_{\text {asd }}^{s}$ классов неприводимых связностей $[a]$. Из неприводимости следует, что $H_{a}^{2}=0$. Тогда $\mathscr{M}_{\text {asd }}^{s}$-ориентируемо. Действительно, как было указано вьше, касательное пространство к многообразию модулей в гладкой точке описывается как ядро эллиптического оператора

$$
\delta_{a}: \Omega^{1}(\operatorname{ad} E) \rightarrow \Omega^{0}(\operatorname{ad} E) \oplus \Omega^{+}(\operatorname{ad} E)
$$

Ориентируемость $\mathscr{M}_{\text {asd }}^{s}$ эквивалентна тривиальности старшей внешней степени касательного расслоения, поэтому необходимо рассмотреть вещественное линейное расслоение $\Lambda^{\max }\left(\operatorname{ker} \delta_{a}\right)$ на $\mathscr{M}_{\text {asd }}^{s}$. Так как коядро coker $\delta_{a}$ тривиально на $\mathscr{M}_{\text {asd }}^{s}$, последнее расслоение отождествляется с детерминантом индексного расслоения оператора $\delta_{a}$, которое представляет собой разность ядра и коядра в $K$-фуннторе. Последнее естественно продолжается на все $\mathscr{B}^{*}$ как детерминант $\delta$, подкрученньй на семейство $\mathrm{SO}(3)$-связностей, приходящих с $\mathrm{ad} E$,

$$
\Lambda=\operatorname{det} \operatorname{ind}(\delta, \operatorname{ad} E) .
$$

Известно, что расслоение $\Lambda$ тривиально, поэтому и существует ориентация на $\mathscr{M}_{\text {asd }}^{s}$. Ориентируемость многообразия модулей имеет место не только для случая $G=$ $\mathrm{SU}(2)$. Утверждение верно и для $\mathrm{SU}(n)$-связностей, а через них, при помощи некоторого трюка, и для $\mathrm{U}(n)$-связностей. Трюк не слишком сложен-вместо $\mathrm{U}(n)$-расслоения 
рассматривается $\mathrm{SU}(n+n)$-расслоение $E_{+}=E \oplus E^{*}$, соответствующее гомоморфизму $\rho: \mathrm{U}(l) \rightarrow \mathrm{SU}(l+l)$, определяемому формулой

$$
\rho(u)=\left(\begin{array}{cc}
u & 0 \\
0 & u^{-1}
\end{array}\right) .
$$

Рассмотрим теперь $\mathrm{SO}(3)$-расслоение $P \rightarrow X$. Так как $X$ - односвязно, существует целочисленный класс $\alpha \in H^{2}(X, \mathbb{Z}), \alpha=w_{2}(P)(\bmod 2)$, такой, что $P$ поднимается до $\mathrm{U}(2)$-расслоения $E \rightarrow X$, причем $c_{1}(E)=\alpha$. Далее, пусть $t: \mathscr{B}_{f, E} \rightarrow \mathscr{B}_{f, P}$ - отображение, порождаемое гомоморфизмом $\mathrm{U}(2) \rightarrow \mathrm{SO}(3)$, и $\Lambda_{E}^{f}, \Lambda_{P}^{f}$ - расслоения ориентаций. Так как слои $t$ связны, то $\Lambda_{P}^{f}$ тривиально, если таковьм является $t^{*}\left(\Lambda_{P}^{f}\right)$. С другой стороны, последнее расслоение изоморфно $\Lambda_{E}^{f}$ (это связано с отождествлением ad $E=\operatorname{ad} P \oplus \mathbb{R})$. Поэтому случай $\mathrm{SO}(3)$ сводится к U(2)-случаю.

Таким образом, имеется результат: в случае общей метрики многообразие модулей неприводимых инстантонов не только гладкое, но и ориентируемое.

С другой стороны, многообразие модулей оснашенных инстантонов может быть получено как результат симплектической редукции. А именно, на пространстве связностей $\mathscr{A}$ имеется естественная симплектическая структура, задаваемая на касательных векторах $u, v \in T \mathscr{A}=\Omega^{1}(\operatorname{ad} E)$ как композиция свертки на $\operatorname{ad} E$ и стандартной симплектической структуры на кокасательном расслоении $T^{*} X$. Отображение моментов $\mu$ задается просто взятием положительной части кривизны (очевидно, такое отображение эквивариантно по действию калибровочной групшы) и $\mathscr{M}_{\text {asd }}=\mu^{-1}(0)$.

\section{§. Компактификация многообразия модулей}

3.1. Идеальные связности. Для нахождения численных инвариантов, связанных с многообразиями модулей инстантонов, необходимо это многообразие компактифицировать. Похожая ситуация возникает при вычислении классов Черна на некомпактном многообразии. А именно, численный результат существенно зависит от компактификации.

Компактификация по Уленбек предполагает определение класса идеальных связностей. Для простоты будем рассматривать случай $\mathrm{SU}(2)$-расслоений. Тогда для ориентированного компактного односвязного риманова четырехмерного многообразия имеется последовательность многообразий модулей $\mathscr{M}_{k}$, занумерованных вторыми классами Черна $k \geqslant 0\left(\mathscr{M}_{0}-\right.$ просто точка, представляюшая тривиальную связность).

Идеальная антиавтодуальная связность класса $k$ определяется как пара

$$
\left([a],\left(x_{1}, \ldots, x_{l}\right)\right)
$$

где $[a] \in \mathscr{M}_{k-l}$ и $\left(x_{1}, \ldots, x_{l}\right)$ - неупорядоченная $l$-ка точек подлежашего многообразия $X$. Плотность кривизны связности $\left([a],\left(x_{1}, \ldots, x_{l}\right)\right)$ определяется как

$$
|F(a)|^{2}+8 \pi^{2} \sum_{r=1}^{l} \delta_{x_{r}} .
$$

Пусть $a_{j}$ - последовательность связностей на $\mathrm{SU}(2)$-расслоении $E_{k}$, второй класс Черна которого равен $k$. Назовем последовательность классов калибровочно 
эквивалентных связностей $\left[a_{j}\right]$ слабо сходящейся к предельной идеальной связности $\left([a],\left(x_{1}, \ldots, x_{l}\right)\right)$, если вьполнены следуюшие условия:

а) для любой непрерьвной на $X$ функции $f$ последовательность значений интегралов $\int_{X} f\left|F\left(a_{j}\right)\right|^{2} d \mu$ сходится:

$$
\int_{X} f\left|F\left(a_{j}\right)\right|^{2} d \mu \rightarrow \int_{X} f|F(a)|^{2} d \mu+8 \pi^{2} \sum_{r=1}^{l} f\left(x_{r}\right)
$$

б) существуют отображения расслоений

$$
\rho_{j}:\left.\left.E_{l}\right|_{X /\left(x_{1}, \ldots, x_{l}\right)} \rightarrow E_{k}\right|_{X /\left(x_{1}, \ldots, x_{l}\right)},
$$

так что последовательность связностей $\rho_{j}^{*}\left(a_{j}\right)$ сходится к $a$.

Для каждого $k$ таким образом определяется топология на множестве всех идеальных связностей

$$
I \mathscr{M}_{k}=\mathscr{M}_{k} \cup\left(\mathscr{M}_{k-1} \times X\right) \cup\left(\mathscr{M}_{k-2} \times S^{2}(X)\right) \cup \cdots .
$$

Многообразие модулей $\mathscr{M}_{k}$ вложено в $I \mathscr{M}_{k}$ как открытое подмножество. Определим тогда $\overline{\mathscr{M}_{k}}$ как замыкание $\mathscr{M}_{k}$ в пространстве всех идеальных связностей $I \mathscr{M}_{k}$.

Справедливо утверждение о том, что любая бесконечная последовательность связностей в $\mathscr{M}_{k}$ имеет слабо сходящуюся подпоследовательность, предел которой содержится в $\overline{\mathscr{M}_{k}}$. Следовательно, $\overline{\mathscr{M}_{k}}$ компактно.

Аналогично и для SO(3)-расслоения при фиксированном классе Штиффеля-Уитни $w \in H^{2}\left(X, \mathbb{Z}_{2}\right)$ имеется многообразие модулей $\mathscr{M}_{k, w}$ связностей на расслоении $E$, причем $k(E)=-\frac{1}{4} p_{1}(E)$, как было определено в п. в) (раздел 2.3). Индекс $k$ на этот раз не обязательно целое число, но разность любых двух индексов обязательно целая. Естественная компактификация в этом случае получается как замыкание $\mathscr{M}_{k, w}$ в

$$
\mathscr{M}_{k, w} \cup\left(\mathscr{M}_{k-1, w} \times X\right) \cup\left(\mathscr{M}_{k-2, w} \times S^{2}(X)\right) \cup \cdots .
$$

Несмотря на ясньй и четкий характер построений, при компактификации могут возникнуть весьма особые точки и локусы. Например, последовательность неприводимых связностей может сходиться к идеальной связности, локально свободная часть которой приводима, и т. д.

3.2. Чудеса начинаются. Всюду далее мы будем рассматривать только односвязные 4-многообразия. В этом случае гомотопический тип многообразия $X$ задается целочисленной квадратичной формой пересечения $q_{X}$ на $H^{2}(X, \mathbb{Z}):$ пусть $\alpha^{*} \in$ $H_{2}(X, \mathbb{Z})$ - двойственный к $\alpha \in H^{2}(X, \mathbb{Z})$ по Пуанкаре цикл, тогда

$$
q_{X}(\alpha, \beta)=\beta\left(\alpha^{*}\right) .
$$

Заметим, что в этом односвязном случае видна четкая параллель со случаем двумерной связной компактной римановой поверхности $\Sigma$ : гомотопический тип $\Sigma$ задается симметрической формой $q_{\Sigma}$ на $H_{1}\left(\Sigma, \mathbb{Z}_{2}\right)$.

$$
q_{\Sigma}\left(\alpha_{1}, \alpha_{2}\right)=\alpha_{1} \cdot \alpha_{2} \quad(\bmod 2) .
$$


В частности, если класс гомологий задан простой замкнутой кривой $C$, то $C^{2}=1$ в том и только том случае, если окрестность кривой $C$ - лист Мёбиуса. Легко видеть, что любая симметрическая форма соответствует некоторой римановой поверхности и однозначно определяет ее гомотопический, топологический и гладкий тип.

Теорема Фридмана [11] гарантирует справедливость этих утверждений и в четырехмерном случае для топологического типа: каждая унимодулярная форма соответствует односвязному ориентированному топологическому многообразию. Если форма четна, то топологическое многообразие одно, а если нечетно, то таких многообразий два.

В отличие от двумерного случая для гладкого 4-многообразия форма пересечения не может быть любой. Первые запреты начинаются с теоремы Рохлина об индексе гладких многообразий с четными формами (см. ниже элементарное доказательство, использующее индексы операторов Дирака). В гладком случае имеется существенная разница между определенными и неопределенными формами. Напомним, что разложение (2.5) определяет два числа $b^{ \pm}(2.6)$. Их сумма $b_{2}=b^{+}+b^{-}$- второе число Бетти нашего многообразия, а их разность $\sigma=b^{+}-b^{-}-$сигнатура нашего многообразия - зависит от ориентации. Определенные формы- это те, у которых либо $b^{+}=0$, либо $b^{-}=0$.

Самое сильное утверждение в гомотопической теории односвязных гладких многообразий содержит следуюшая теорема Дональдсона.

Теорема 3.1. Определенная форма гладкого односвязного 4-многообразия $X$ является челочисленной суммой квадратов.

Иначе говоря, гомотопический тип такого многообразия с точностью до ориентации определяется вторым числом Бетти.

Доказательство этой теоремы рекордно короткое (опять же по модулю всей теории Дональдсона). Пусть наша форма $q_{X}$ отрицательно определена. Рассмотрим для общей метрики на $X$ многообразие $\mathscr{M}_{\mathrm{asd}}(1)$ модулей $\mathrm{SU}(2)$-инстантонов с $c_{2}=1$ (называемых обычно 1-инстантонами). Согласно результатам раздела $2.4 \mathscr{M}_{\text {asd }}(1)$ - либо гладкое пятимерное многообразие (см. формулу (2.33)) вне конечного числа простых особых точек, соответствуюших приводимым связностям, либо пусто. Каждая приводимая связность соответствует разложению расслоения $E=L \oplus L^{*}$ и связности $a=a_{L} \oplus a_{L^{*}}$, где $a_{L}$ - антиавтодуальная абелева связность. Но поскольку $c_{2}(E)=1$, то $c_{1}(L)^{2}=-1$. Далее, компактификация $\overline{\mathscr{M}_{\text {asd }}(1)}$ содержит идеальные связности вида $([a], x)$ (см. $(3.1)$ и $(3.6))$, где $[a]$ - тривиальная плоская связность на тривиальном расслоении, а $x$ - любая точка нашего многообразия $X$. Такие связности, конечно, сушествуют и являются идеальными антиавтодуальными связностями. Локальное многообразие деформаций таких решений пятимерно и содержит неприводимые антиавтодуальные связности. Значит,

(1) пятимерное пространство $\mathscr{M}_{\text {asd }}(1)$ не пусто;

(2) компактификация $\overline{\mathscr{M}_{\text {asd }}(1)}$ доставляет гладкий ориентированньй бордизм между $X$ и несвязным объединением линков особых точек - приводимых связностей;

(3) линк каждой особой точки есть $\mathbb{C P}^{2}$ с обращенной ориентацией.

Сильнейшая теорема гомотопической теории гладких 4-многообразий доказана.

Перейдем теперь к случаю неопределенных форм: если форма $q_{X}$ нечетная, то она 
представима в виде

$$
q_{X}=\sum_{1}^{b^{+}} x_{i}^{2}-\sum_{1}^{b^{-}} y_{j}^{2}
$$

а если четная, то она имеет вид

$$
q_{X}=2 k E_{8} \oplus m H
$$

где $E_{8}$ - определенная стандартная форма ранга $8, H$ - стандартная гиперболическая форма ранга 2 , а коэффициент при $E_{8}$ четный по теореме Рохлина. Этот результат показывает, что нечетные формы реализуются все в качестве форм пересечений гладких многообразий. Для четных форм проблема открыта при $k \geqslant 1$. Результаты, имеющиеся на сегодняшний день в этой задаче, следуюшие:

(1) Дональдсон (1984 г.)

$$
k \geqslant 1 \Longrightarrow m \geqslant 3
$$

(2) Кронхеймер-Мровка (1994 г.)

$$
k \geqslant 2 \Longrightarrow m \geqslant 6
$$

$$
k \geqslant 3 \Longrightarrow m \geqslant 9 .
$$

Заметим, что для К3-поверхности $k=1, m=3$ и, значит, связные суммы К3-поверхностей и квадрик реализуют все формы при $m \geqslant 3 k$.

Последний шаг гомотопической классификации гладких 4-многообразий -

$\frac{11}{8}$-ГИПОТЕЗА: $3 k \leqslant m$.

Заметим, что Фурута анонсировал решение проблемы “ $\frac{10}{8}$ ” и что для 4-многообразий, подлежаших алгебраическим поверхностям, гипотеза следует из неравенства Богомолова-Мияока-Яо (см., например, [22]). Геометрическое следствие гипотезы существование топомодели любого односвязного гладкого 4-многообразия:

$$
\begin{aligned}
q_{X} \text { - нечетна } & \Longrightarrow X \sim b^{+} \mathbb{C P}^{2} \# b^{-\overline{\mathbb{C P}^{2}}}, \\
q_{X}-\text { четна } & \Longrightarrow X \sim \pm \frac{\sigma}{16} K \#(m-3 k) Q,
\end{aligned}
$$

где $\sim$ - топологическая эквивалентность, \# - операция связной суммы, $\overline{\mathbb{C P}^{2}}$ комплексная проективная плоскость с обращенной ориентацией, $\pm K-\mathrm{K} 3$-поверхность со стандартной или обращенной ориентацией и $Q-$ комплексная квадрика, $Q=$ $\mathbb{C} \mathbb{P}^{1} \times \mathbb{C P}^{1}$.

Мы видим, что, как и в двумерном случае, каждое гладкое односвязное 4-многообразие может быть представлено топологически как связная сумма проективных плоскостей, “торов” (т.е. произведений сфер соответствуюшей размерности) и кватернионных эллиптических кривых - К3-поверхностей (с точностью до ориентации). Однако гладкая классификация гораздо богаче: не всякая алгебраическая поверхность гладко эквивалентна своей топомодели. Замечательньй результат заключается в том, что для алгебраических поверхностей с $b^{+}>1$ гладкая структура ее топомодели является экзотической, т.е. не совпадает с ее подлежащей гладкой структурой.

Здесь мы уже прочно привязаны к опыту алгебраической геометрии (см., например, $[21])$, для которой описанные в следующем разделе конструкции в их алгебро-геометрическом варианте давно уже стали привьчньми (если не сказать “банальньми”) (см. оригинальную работу Мукая [14]). 
3.3. $\mu$-классы. Основная идея следующих построений заключается в том, что когомологические классы множества, параметризующего орбиты в семействе связностей, могут быть подняты с подлежащего многообразия.

А именно, мы будем искать гомологические классы пространств $\mathscr{B}$ и $\mathscr{B}^{*}$.

Отметим, что при изучении топологических свойств этих пространств трудности могут возникнуть из несвободы действия калибровочной группы на пространстве связностей. Даже в случае неприводимых связностей стабилизатор $\Gamma_{a}$ может быть нетривиальным - равным центру $C(G)$. Поэтому обычно удобно работать с оснашенными связностями.

Пусть на подлежащем многообразии отмечена некоторая точка $x_{0}$. Тогда оснащенной связностью на главном расслоении $P$ назьвается пара $(a, f)$, где $a-$ связность, а $f$ - эквивариантный по действию $G$ изоморфизм $\phi: G \rightarrow P_{x_{0}}$. В наших рассмотрениях это равносильно заданию ортонормированного базиса в слое расслоения $E_{x_{0}}$. Калибровочная группа естественно действует на пространстве оснашенных связностей, поэтому можно определить

$$
\mathscr{B}_{f}=\left(\mathscr{A} \times \operatorname{Hom}\left(G, P_{x_{0}}\right)\right) / \mathscr{G}
$$

Другой способ определения $\mathscr{B}_{f}$ - рассмотрение подгруппы калибровочной групшы $\mathscr{G}_{0} \subset \mathscr{G}$ такой, что

$$
\mathscr{G}_{0}=\left\{u \in \mathscr{G} \mid u\left(x_{0}\right)=\mathrm{id}\right\} .
$$

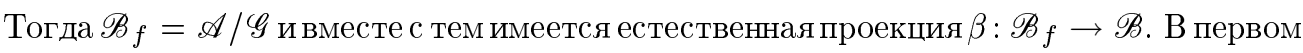
определении $\beta$ - отображение, забьвающее фиксированньй в точке $x_{0}$ базис.

Стабилизатор $\Gamma_{a}$ состоит из ковариантно-постоянных преобразований, подгруппа $\mathscr{G}_{0}$ действует свободно на $\mathscr{A}$, следовательно, $\mathscr{B}_{f}$ банахово многообразие. Слой отображения $\beta$ в точке $[a]$ изоморфен $G / \Gamma_{a}$, где $\Gamma_{a}$ понимается как подгруппа $G$ через отождествление $\mathscr{G} / \mathscr{G}_{0} \cong G$.

Построим семейство оснашенных связностей, параметризованное $\mathscr{B}_{f}$. Пусть $\pi_{2}: \mathscr{A} \times X \rightarrow X-$ проекция на второй сомножитель, тогда $P^{\prime} \rightarrow \mathscr{A} \times X$ определяется как поднятие $\pi_{2}^{*}(P)$. Поэтому $P^{\prime}=\mathscr{A} \times P$. Тогда на $P^{\prime}$ поднимается семейство связностей $\mathscr{A}^{\prime}$, так что на срезе $P_{a}$ над $a \times X$ связность совпадает с $\pi_{2}^{*}(a)$. Если выбор базиса $f$ в $x_{0}$ сделан, тогда мы получаем оснашенность и для всего семейства $f^{\prime}$. Группа $\mathscr{G}_{0}$ действует свободно на $\mathscr{A} \times X$, так же как и на $P^{\prime}$, поэтому можно говорить о фактор-расслоении

$$
\begin{gathered}
\mathbb{P}_{f} \rightarrow \mathscr{B}_{f} \times X, \\
\mathbb{P}_{f}=P^{\prime} / \mathscr{G}_{0} .
\end{gathered}
$$

Семейства связностей $\mathscr{A}^{\prime}$ и $f^{\prime}$ сохраняются при действии $\mathscr{G}_{0}$, поэтому фактор-расслоение (3.17) наследует семейство базисных связностей $\left(\mathbb{A}^{\prime}, \mho\right)$.

Пространство $\mathscr{B}^{*}$ не параметризует никакое универсальное семейство. Но можно действовать так: пусть $\mathscr{A}^{*} \subset \mathscr{A}$ - подпространство неприводимых связностей; тогда можно определить $P^{\prime} \rightarrow \mathscr{A}^{*} \times X$ как поднятие расслоения $P^{\prime}=\mathscr{A}^{*} \times P$. Как и выше, можно поднять семейство связностей. Калибровочная группа действует на этом семействе, но не действует свободно на базе $\mathscr{A}^{*} \times X$, кроме случая, когда центр групшы 
тривиален. Так как $C(G)$ действует тривиально на базе и нетривиально на рассло-

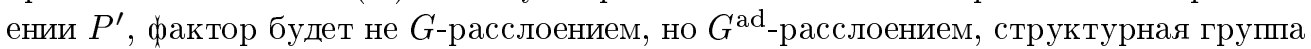
которого есть $G / C(G)$. И возможно определить

$$
\mathbb{P}^{\text {ad }} \rightarrow \mathscr{B}^{*} \times X
$$

как фактор $\mathbb{P}^{\text {ad }}=P^{\prime} / \mathscr{G}$.

В наших рассмотрениях, когда $G=\mathrm{SU}(2), \mathbb{P}^{\mathrm{ad}}$ будет $\mathrm{SO}(3)$-расслоением над $\mathscr{B}^{*} \times X$.

Чтобы описать в этом случае когомологии $\mathscr{B}_{f}$ и $\mathscr{B}^{*}$, необходимо использовать естественное произведение

$$
/: H^{d}\left(\mathscr{B}_{f} \times X\right) \times H_{j}(X) \rightarrow H^{d-j}\left(\mathscr{B}_{f}\right) .
$$

(Заметим, что символ / - стандартное обозначение slant-произведения.) Для любого характеристического класса $c$, связанного с группой $G$, сушествует класс когомологий $c\left(\mathbb{P}_{f}\right) \in H^{d}\left(\mathscr{B}_{f} \times X\right)$, где $d=\operatorname{deg}(c)$. И отображение определяется как "свертка"

$$
\begin{gathered}
\mu_{c}^{f}: H_{j}(X) \rightarrow H^{d-j}\left(\mathscr{B}_{f}\right), \\
\mu_{c}^{f}(\alpha)=c\left(\mathbb{P}_{f}\right) / \alpha .
\end{gathered}
$$

Сходньм образом определяются классы на $\mathscr{B}^{*}$, но на этот раз берется характеристический класс групшы $G^{\mathrm{ad}}$, и отображение

$$
\begin{gathered}
\mu_{c}: H_{j}(X) \rightarrow H^{d-j}\left(\mathscr{B}^{*}\right), \\
\mu_{c}(\alpha)=c\left(\mathbb{P}^{\mathrm{ad}}\right) / \alpha
\end{gathered}
$$

задает классы когомологий факторпространства неприводимых связностей.

Продолжим рассуждения определением $\mu$-классов в нашем конкретном случае. Для главного $\mathrm{SU}(2)$-расслоения отображение $\mu^{f}: H_{2}(X, \mathbb{Z}) \rightarrow H^{2}\left(\mathscr{B}_{f}, \mathbb{Z}\right)$ задается формулой

$$
\mu^{f}(\Sigma)=c_{2}\left(\mathbb{P}_{f}\right) /[\Sigma],
$$

отображение $\mu: H_{2}(X, \mathbb{Q}) \rightarrow H^{2}\left(\mathscr{B}^{*}, \mathbb{Q}\right)$ задается сходным образом:

$$
\mu(\Sigma)=-\frac{1}{4} p_{1}\left(\mathbb{P}^{\mathrm{ad}}\right) /[\Sigma]
$$

Второе из этих определений подходит и тогда, когда $G=\mathrm{SO}(3)$; в каждом из случаев $\mathbb{P}^{\text {ad }}$ является SO(3)-расслоением.

Определения, представленные вьше, дают отображения средних когомологий подлежашего многообразия. Кратко опишем случаи $\mu_{c_{2}}^{f}: H_{j}(X) \rightarrow H^{4-j}\left(\mathscr{B}^{*}\right)$, когда $j=1$ и 3. Каждьй из них имеет прямую геометрическую интерпретацию.

Пусть $\gamma$ - замкнутый путь, начинаюшийся и заканчиваюшийся в $x_{0}$ и представляюший класс $[\gamma] \in H^{1}(X, \mathbb{Z})$. Для любой связности $a$ обозначим через $h_{\gamma}(a)$ голономию связности вдоль замкнутого контура. Это автоморфизм слоя $P_{x_{0}}$, зависящий от класса эквивалентности $a$ как отмеченной связности и определяюший отображение

$$
h_{\gamma}: \mathscr{B}_{f}=\left(\mathscr{A} / \mathscr{G}_{0}\right) \rightarrow \mathrm{SU}(2) \cong S^{3} .
$$


Следовательно, мы получаем класс когомологий $h_{\gamma}^{*}(\omega) \in H^{3}\left(\mathscr{B}_{f}\right)$ поднятием фуундаментального класса $\omega \in H^{3}\left(S^{3}\right)$, и нетрудно доказать, что этот класс когомологий совпадает с $\mu_{c_{2}}^{f}([\gamma])$.

Теперь рассмотрим отображение $\mu_{c_{2}}^{f}: H^{3}(X) \rightarrow H^{1}\left(\mathscr{B}_{f}\right)$. Если $[Y] \in H^{3}(X)-$ класс, представителем которого является вложенное 3 -многообразие $Y \subset X$, тогда для любой связности $a$ на $X$ можно вычислить инвариант Черна-Саймонса для ограничения $\left.a\right|_{Y}$. Этот инвариант $\tau_{Y}(a)$ принимает значения в $S^{1}$ и, следовательно, определяет отображение $\tau_{Y}: \mathscr{B}_{f} \rightarrow S^{1}$. И снова из фундаментального класса, но теперь уже $S^{1}$, при поднятии получаем элемент из $H^{1}\left(\mathscr{B}_{f}\right)$. Тождественность этого класса с $\mu_{c_{2}}^{f}([Y])$ легко вьводится из определения второго класса Черна через кривизну в теории Черна-Вейля.

Но вернемся к двумерным когомологиям. В случае односвязного многообразия образ отображения, определенного формулой (3.20), порождает все рациональные когомологии $\mathscr{B}_{f}$. Вообше верен следуюший факт: если $P$ - главное $\mathrm{SU}(2)$-расслоение над односвязньм 4-многообразием и $\Sigma_{1}, \ldots, \Sigma_{b_{2}}$ - базис в $H_{2}(X, \mathbb{Z})$, тогда кольцо рациональных когомологий $H^{*}\left(\mathscr{B}_{f}, \mathbb{Q}\right)$ есть полиномиальная алгебра на образуюших $\mu^{f}\left(\Sigma_{1}\right), \ldots, \mu^{f}\left(\Sigma_{b_{2}}\right)$. В частности, $H^{2 k}\left(\mathscr{B}_{f}, \mathbb{Q}\right) \cong S^{k}\left(H_{2}(X, \mathbb{Q})\right)$.

Этот результат может быть использован для вычислений рациональных когомологий $\mathscr{B}^{*}$ при помоши поднятия, задаваемого отображением $\beta$. Класс $\mu(\Sigma) \in H^{2}\left(\mathscr{B}^{*}, \mathbb{Q}\right)$ поднимается до класса $\mu^{f}(\Sigma)$, но в этом случае данные классы не являются образуюшими кольца когомологий базы. Необходимо еще добавить 4-мерный класс

$$
\nu=-\frac{1}{4} p_{1}(\beta) \in H^{4}\left(\mathscr{B}^{*}, \mathbb{Q}\right)
$$

где $p_{1}(\beta)$ - класс Понтрягина $\beta$ как $\mathrm{SO}(3)$-расслоения. Это определение может быть рассмотрено как пример общей конструкции $\mu_{c}(\alpha)$ в случае, когда $c=-\frac{1}{4} p_{1}$ и $\alpha=$ $\left[x_{0}\right] \in H_{0}(X)$.

Сходное утверждение формулируется для кольца когомологий $H^{*}\left(\mathscr{B}^{*}, \mathbb{Q}\right)$, а именно, это полиномиальная алгебра на образуюших - одном четырехмерном и $b_{2}$ двумерных:

$$
H^{*}\left(\mathscr{B}^{*}, \mathbb{Q}\right)=\mathbb{Q}\left[\nu, \mu\left(\Sigma_{1}\right), \ldots, \mu\left(\Sigma_{b_{2}}\right)\right] .
$$

Рассмотрим следуюший пример. Пусть в качестве подлежащего многообразия взята риманова поверхность $S$, и $G=\mathrm{U}(1)$. Пространства $\mathscr{B}_{f}, \mathscr{B}, \mathscr{B}^{*}$ все совпадают в этом случае. Так как в случае комплексной размерности 1 нет условий интегрируемости, любая связность на линейном расслоении $L \rightarrow S$ задает голоморфную структуру на $L$. Это определяет отображение $\mathscr{B} \rightarrow \mathrm{Pic}_{k}(S)$, где $\mathrm{Pic}_{k}$ - торы классов изоморфных голоморфных линейных расслоений степени $k$. Такое отображение сохраняет гомотопическую эквивалентность, слои его - пространство всех эрмитовых метрик на $L$. Следовательно, когомологии $\mathscr{B}$ те же, что и на якобиане, а так как последний есть $2 g$-тор, то $H^{*}(\mathscr{B}, \mathbb{Z})$ - внешняя алгебра с $2 g$ одномерньми образуюшими. Отождествив их с классами $\mu_{c_{1}}(\alpha), \alpha \in H_{1}(S)$, получаем обычное описание якобиана как фактора $\mathbb{R}^{2 g}$ по решетке периодов.

3.4. Инварианты Дональдсона. Как всегда, начнем с простого случая - случая нульмерного многообразия модулей инстантонов. 
Пусть $X$ - односвязное 4-многообразие с $b^{+}(X)>1$ (условие, устраняющее зависимость от выбора метрики), и пусть $E \rightarrow X$ - расслоение со структурной группой $G=\mathrm{SO}(3)$. Пусть виртуальная размерность многообразия модулей равна нулю: $8 k-3\left(1+b^{+}\right)=0$. Тогда для общей метрики $g$ верны следуюшие утверждения:

(1) многообразие модулей $\mathscr{M}_{\text {asd }}(E, g)$ содержит только неприводимые связности;

$(2) \mathscr{M}_{\text {asd }}(E, g)$ состоит из конечного числа точек;

(3) выбирая ориентацию, можно присвоить знак каждой точке $\mathscr{M}_{\text {asd }}(E, g)$.

Выбор ориентации зависит от выбора ее на расслоении $\Lambda=\operatorname{det} \operatorname{ind}(\delta, \operatorname{ad} E)$, однако такой выбор становится каноническим, если зафиксировать ориентацию на $H^{+}\left(X_{0}\right)$ и класс эквивалентности целочисленного подъема $w_{2}(E)$ в случае $\mathrm{SO}(3)$-расслоения.

$\mathscr{M}_{\text {asd }}(E, g)$ в этом случае, очевидно, компактно, так как при меньших $k$ антиавтодуальных связностей нет, а значит, нет и предельных идеальных связностей, все пределы - действительные. Это дает компактность $\mathscr{M}_{\text {asd }}(E, g)$, равносильную конечности числа точек.

Определим инвариант как целое число

$$
q=\sum_{[a] \in \mathscr{M}} \varepsilon(a)
$$

A priori это число зависит от метрики, выбора ориентации на $H^{+}(X)$ и целочисленного подъема.

Замечательное свойство такого “числа точек многообразия модулей, взятых со знаками", заключается в том, что для двух обших метрик $g_{0}$ и $g_{1}$ имеем равенство

$$
q\left(g_{0}\right)=q\left(g_{1}\right)
$$

В качестве примера рассмотрим К3-поверхность. Пусть

$$
\alpha \in H^{2}\left(X, \mathbb{Z}_{2}\right), \quad \alpha^{2}=2 \quad(\bmod 4) .
$$

Рассмотрим $\mathrm{SO}(3)$-расслоение $F$ такое, что $w_{2}(F)=\alpha$ и $p_{1}(F)=-6$.

Так как для К3-поверхности $b^{+}(X)=3$, виртуальная размерность многообразия модулей равна

$$
\operatorname{dim} \mathscr{M}_{F}=-2 p_{1}(F)-3(1+3)=0 .
$$

Значит, мы находимся в ситуации, когда можно определить инвариант $X$. Для этого необходимо выбрать ориентацию $\Omega$ положительной части $H^{2}(X)$. Форма пересечения К3-поверхности четна, поэтому зависимости от подъема $\alpha$ нет. Запишем инвариант как $q(\alpha, \Omega)$.

Верно следуюшее утверждение: существуют такие $\alpha$ и $\Omega$, что $q(\alpha, \Omega)=1$.

Следствия представляют интересные результаты для К3-поверхностей:

(1) Не сушествует диффеоморфизма, действуюшего тривиально на $H^{2}\left(X, \mathbb{Z}_{2}\right)$, но обрашающего ориентацию на положительной части $H^{2}(X, \mathbb{R})$. (То есть комплексная ориентация К3-поверхности определяет и ее спинорную ориентацию.)

(2) Существует односвязный пятимерный $h$-кобордизм К3-поверхности, не являющийся прямым произведением.

(3) Не сушествует разложения в связную сумму $X=Y \#\left(S^{2} \times S^{2}\right)$. 
Теперь обратимся к более общей ситуации.

Пусть теперь $E \rightarrow X-$ расслоение со структурной группой $\mathrm{SU}(2)$. Инварианты будут представляться в виде пересечений $\left\langle\beta,\left[\mathscr{M}_{k}\right]\right\rangle$, где $\beta$ - результат пересечений классов вида $\mu(\sigma)$, а $\left[\mathscr{M}_{k}\right]$ - фундаментальный класс многообразия модулей инстантонов. Так как классы $\mu(\sigma)$ двумерны, необходимо потребовать, чтобы виртуальная размерность $\mathscr{M}_{k}$ была четна: $\operatorname{dim} \mathscr{M}_{k}=2 d$,

$$
d=d(k)=4 k-\frac{3}{2}\left(b^{+}(X)+1\right) \in \mathbb{Z}
$$

Как и вьше, потребуем $b^{+}>1$ и добавим, исходя из (3.30), условие нечетности.

Пусть $\left[\Sigma_{1}\right], \ldots,\left[\Sigma_{d}\right]-$ классы в $H_{2}(X, \mathbb{Z})$, тогда $\mu\left(\Sigma_{i}\right)$ - поднятия их в $H^{2}\left(\mathscr{B}^{*}, \mathbb{Z}\right)$. Их произведение имеет степень $2 d$, поэтому можно определить целое число

$$
q=\left\langle\mu\left(\Sigma_{1}\right) \smile \cdots \smile \mu\left(\Sigma_{d}\right),\left[\mathscr{M}_{k}\right]\right\rangle
$$

Ориентация $\mathscr{M}_{k}$ зависит от ориентации $\Omega$ пространства $H^{+}(X)$, поэтому мы должны обозначать инварианты как $q_{k}, \Omega$. Тогда запишем свойства инвариантов:

(1) $q_{k, \Omega}\left(\Sigma_{1}, \ldots, \Sigma_{d}\right)$ зависят от аргументов только как от классов гомологий;

(2) $q_{k, \Omega}\left(\Sigma_{1}, \ldots, \Sigma_{d}\right)$ полилинейны и симметричны по $\left[\Sigma_{i}\right]$;

(3) $q_{k, \Omega}=-q_{k,-\Omega}$;

(4) если $f: X \rightarrow Y$ - сохраняющий ориентацию диффеоморфизм, тогда

$$
q_{k, f * \Omega}\left(f\left(\Sigma_{1}\right), \ldots, f\left(\Sigma_{d}\right)\right)=q_{k, \Omega}\left(\Sigma_{1}, \ldots, \Sigma_{d}\right) .
$$

Такие инварианты строятся для достаточно больших $k$. Точное условие на величину $k$ следуюшее:

$$
d(k) \geqslant 2 k+1
$$

или, то же самое, $k \geqslant \frac{1}{4}\left(3 b^{+}(X)+5\right)$. Это ограничение объясняется просто: виртуальная размерность многообразия модулей инстантонов превьшает размерность наименьшего страта $\mathscr{M}_{0} \times S^{k}(X)$ компактификации $\overline{\mathscr{M}_{k}}$ по меньшей мере на два, а это означает, что $\overline{\mathscr{M}}_{k}$ в общем случае гладкое многообразие, за исключением множества коразмерности два или более, что и обычно для особенностей комплекса, обладающего фундаментальным классом.

С помошью этих новых инвариантов возможно доказывать старые гипотезы, передоказывать старые теоремы и получать новые. Например, Дональдсон доказал с их помощью следующее утверждение.

ТеОрема 3.2. Пусть $X$ - односвязное 4-многообразие с нечетным $b^{+}(X), u$ предположим, что $X$ раскладьввается в связную сумму $X=X_{1} \# X_{2}$, причем $b^{+}\left(X_{i}\right)>0, i=1,2$. Тогда $\mathrm{SU}(2)$-инварианты $q_{k}$ u $\mathrm{SO}(3)$-инварианты $q_{k, \alpha}$ (для любых $k$ и $\alpha \neq 0$ ) тождественно равны нулю.

Этот результат не имел бы смысла, если бы инварианты Дональдсона были тривиальны почти всегда. Но для алгебраических и симплектических многообразий они нетривиальны, поэтому использование теории Дональдсона в современной математике приводит к возрождению интереса к алгебраической геометрии: все топомодели 
алгебраических поверхностей (кроме элементарных) допускают вьшеописанное разложение, а значит, топомодели не диффеоморфны подлежашим 4-многообразиям алгебраических поверхностей.

С другой стороны, исследование гладкостей подчеркивает близость комплексных и симплектических структур, и Дональдсон далеко продвинулся в описании этой “близости" (см. [4], [5]).

Столь принципиальное отличие дифференциальной топологии от топологии в четырехмерном случае на некоторое время привело в замешательство и физиков. Причем это коснулось двух диаметрально противоположных областей: традиционно элитарной квантовой гравитации (инициированной Тулио Редже) и сугубо практических вычислений на решетках с использованием метода Монте-Карло (см., например, [1]). Все дело в том, что комбинаторные конструкции такие, как, например, метод динамической триангуляции, используют PL-топологию 4-многообразий. A, как известно, PL-структуры и гладкости в четырехмерной геометрии совпадают. От этого временного замешательства вьпграли математики: они немедленно применили хорошо известные статистические приемы к совокупности инвариантов $q_{k, \Omega}(3.31)$. Идея проста: собрать все инварианты вместе в "статсумму"

$$
q_{\Omega}=\sum_{0}^{\infty} \frac{q_{k, \Omega}}{k !} .
$$

Подобно тому, как в теории динамических триангулящй аналитика статсумм приводит к нахождению и локализации критических точек, аналитика функции (3.34) привела Кронхеймера и Мровку к замечательному наблюдению: при естественных условиях на 4-многообразие $X$ (так назьваемая "простота типа" подлежащего многообразия) существует конечное множество классов когомологий

$$
\left\{k_{1}, \ldots, k_{N}\right\}_{K M} \subset H^{2}(X, \mathbb{Z})
$$

таких, что каждый полином Дональдсона содержится в рациональной оболочке

$$
q_{k, \Omega} \in \mathbb{Q}\left[q_{X}, k_{1}, \ldots, k_{N}\right]
$$

(здесь форма пересечений $q_{X}$ рассматривается как квадратичная форма на решетке $H^{2}(X, \mathbb{Z})$, а классы $k_{i}$ - как линейные формы). А вся статсумма имеет очень простой вид

$$
q_{\Omega}=\exp \left(\frac{q_{X}}{2}\right) \sum_{i=1}^{N} n_{i} \exp \left(k_{i}\right) .
$$

Эти классы были названы фундаментальными классами, и читатель-физик без сомнения заметит, что они являются аналогами фаз сильного, слабого взаимодействий и "полимеризации" для асимптотик динамических триангулящий.

Эти классы действительно фундаментальны и играют роль канонического класса алгебраической поверхности для гладкого случая. В частности, как линейньй член они входят в формулу присоединения для оценки рода реализации класса 2-гомологий гладкой римановой поверхностью (см. ниже).

Здесь мы отметим пока только одно их свойство: множество (3.35) инвариантно относительно обрашения знака, т.е. если класс $k_{i}$ фундаментальньй, то и класс 
$-k_{i}=k_{-i}$ тоже фундаментальный. Более того, константа $n_{-i}=(-1)^{\frac{b^{+}+1}{2}}$. Поэтому, рассматривая формальные переменные $t_{i}=\exp \left(k_{i}\right)$ и убирая из статсуммы $(3.37)$ топологический множитель $\exp \left(q_{X} / 2\right)$, мы получаем полином Лорана

$$
K M_{X}=n_{0}+\sum n_{i}\left(t_{i}+(-1)^{\frac{b^{+}+1}{2}} t_{i}^{-1}\right)
$$

которьй (как мы увидим ниже) не случайно напоминает полином Александера линка в $S^{3}$.

В частности, если переменная только одна, то $K M_{X}$ является А-полиномом: $n_{0}+$ $\sum_{i=1}^{N} n_{i}\left(t^{i}+t^{-i}\right)$ и $n_{0}+\sum_{i=1}^{N} n_{i}= \pm 1$. Напомним, что любой такой полином является полиномом Александера узла в $S^{3}$. Мы обсудим эти “совпадения" в $\S 6$ этого обзора.

Наконец, заметим, что конфигурация теории Янга-Миллса (как классической теории поля) слишком проста, несмотря на блестяшие физические приложения (отмеченные двумя нобелевскими премиями). Выбор более сложных конфигураций приводит к следуюшему этапу - теории Зайберга-Виттена.

\section{$\S$ 4. Инварианты Зайберга-Виттена}

4.1. Алгебры Клиффорда. Для произвольного $n$-мерного вешественного векторного пространства $V$ с невырожденной квадратичной формой $q$ алгеброй Клифифорда $C(V, q)$ называется фактор тензорной алгебры $T(V)$ по идеалу, содержашему элементы вида $v \otimes v-q(v) \cdot 1$. Универсальность алгебры $C(V, q)$ заключается в том, что если существует какое-либо линейное отображение пространства $V$ в некоторую алгебру $K$, причем форма при этом сохраняется (т.е. образ любого вектора, возведенньй в квадрат, должен быть равен значению формы на этом векторе, умноженному на единицу алгебры), то это отображение пропускается через каноническое вложение пространства $V$ в алгебру Клиффорда $C(V, q)$.

Очевидно, что если выбрать базис в $V$, например, канонический для формы $q$, тогда алгебра Клиффорда задается соотношениями

$$
\begin{gathered}
e_{j}^{2}=-1, \\
e_{i} \cdot e_{j}=-e_{j} \cdot e_{i} .
\end{gathered}
$$

Базисом алгебры как вещественного векторного пространства будут мономы вида

$$
\begin{gathered}
e_{I}=e_{i_{1}} \cdot \ldots \cdot e_{i_{k}}, \\
\left\{i_{1}, \ldots, i_{k}\right\} \subset\{1, \ldots, n\},
\end{gathered}
$$

причем наборы строго упорядочены. Число $k$ обьчно назьвается степенью монома.

Очевидно, размерность алгебры равна $2^{n}$, и имеется $\mathbb{Z}_{2}$-градуировка, отвечающая разложению на мономы четной и нечетной степени $C^{+}$и $C^{-}$, причем $C^{+}-$подалгебра в алгебре Клиффорда.

В случае четного $n$ в центре алгебры лежат только константы. В нечетном случае добавляются элементы максимальной степени. 
Алгебра Клиффорда допускает подъем скалярного произведения, определяемого формой $q$, а именно, если элементы алгебры $x$ и $y$ имеют в каноническом базисе координаты $x_{I}$ и $y_{I}$ соответственно, тогда произведение представляется естественно как

$$
(x, y)=\sum_{I} x_{I} \cdot y_{I}
$$

Кроме того, существует еще и естественная инволюция $C(V) \rightarrow C(V)$, определяемая по формуле

$$
\begin{aligned}
& \bar{x}=\sum_{I} \varepsilon_{I} \cdot x_{I} \cdot e_{I}, \\
& \varepsilon_{I}=(-1)^{\frac{1}{2} k(k+1)},
\end{aligned}
$$

где $|I|=k$, причем нетрудно показать, что $\overline{x \cdot y}=\bar{y} \cdot \bar{x}$. Несмотря на определение в терминах некоторого базиса, ни внешнее произведение, ни инволюция от выбора базиса не зависят. Они связаны следуюшим соотношением:

$$
(\bar{x} \cdot y)_{0}=(x, y)
$$

Для доказательства достаточно заметить, что $e_{I}^{2}=\varepsilon_{I}$.

Вообще классификация алгебр Клиффорда достаточно проста. Рассмотрим сначала несколько примеров. Мы будем обозначать парой нижних индексов сигнатуру формы. Тогда для вещественного случая имеем:

(1) $C\left(\mathbb{R}, q_{0,1}\right)=\mathbb{C}$, а если сигнатура формы имеет обратный знак, то

$$
C\left(\mathbb{R}, q_{1,0}\right)=\mathbb{R} \oplus \mathbb{R}
$$

(2) $C\left(\mathbb{R}^{2}, q_{0,2}\right)=\mathbb{H}$, так как порождается элементами $e_{1}, e_{2}$ и $e_{1} \cdot e_{2}$, умножающимися по тем же правилам, что и $i, j, k$; в двух других случаях $C\left(\mathbb{R}^{2}, q\right)$ есть просто алгебра матриц $\operatorname{Mat}_{2}(\mathbb{R}) ;$

(3) известны рекуррентные соотношения

$$
\begin{aligned}
& C\left(\mathbb{R}^{2}, q_{1,1}\right) \otimes C\left(\mathbb{R}^{l+m}, q_{l, m}\right)=C\left(\mathbb{R}^{l+m+2}, q_{l+1, m+1}\right), \\
& C\left(\mathbb{R}^{2}, q_{2,0}\right) \otimes C\left(\mathbb{R}^{l+m}, q_{l, m}\right)=C\left(\mathbb{R}^{l+m+2}, q_{m+2, l}\right), \\
& C\left(\mathbb{R}^{2}, q_{0,2}\right) \otimes C\left(\mathbb{R}^{l+m}, q_{l, m}\right)=C\left(\mathbb{R}^{l+m+2}, q_{m, l+2}\right),
\end{aligned}
$$

которые несложно вьвести, рассматривая все в канонических базисах.

Из соотношений (4.6) следует, что любая алгебра Клиффорда для квадратичной формы максимального ранга является либо матричной алгеброй над $\mathbb{R}, \mathbb{C}$ или $\mathbb{H}$, либо прямой суммой двух одинаковых матричных алгебр.

В случае комплексного векторного пространства классификащия еще более простая - если размерность пространства четна, тогда алгебра Клиффорда изоморфна матричной алгебре $\operatorname{Mat}_{2} \frac{1}{2} \operatorname{dim} V(\mathbb{C})$, в нечетном случае - прямой сумме двух таких алгебр.

Элемент алгебры Клиффорда $x \in C(V)$ назьвается единицей, если существует обратньй ему $x^{-1}$. 
Обозначим через $\operatorname{Spin}(V)$ множество элементов алгебры, удовлетворяюших соотношениям

$$
\bar{x} \cdot x=1, \quad x \cdot V \cdot \bar{x}=V, \quad x \in C^{+}(V) .
$$

Если размерность $V$ четна, то третье условие является следствием первых двух.

Эта конструкция позволяет построить двулистное нетривиальное накрытие группы $\mathrm{SO}(n)$. Если $\operatorname{dim} V$ больше двух, тогда группа $\operatorname{Spin}(V)$ компактна и односвязна, и гомоморфизм $\mathrm{Ad}: \operatorname{Spin}(V) \rightarrow \mathrm{SO}(V)$ задается присоединенным действием $x \in$ $\operatorname{Spin}(V)$ на $V$, а именно,

$$
\operatorname{Ad}(x)(v)=x \cdot v \cdot \bar{x} .
$$

Доказательство этого факта основывается на рассмотрении алгебр Ли обеих груп, причем

$$
\operatorname{Lie}(\operatorname{Spin}(V))=C_{2}(V),
$$

где $C_{2}(V)$ - подмножество элементов алгебры Клиффорда, имеющих вторую степень. Гомоморфизм аd: $C_{2} \rightarrow \mathfrak{s o}(V)$, задаваемьй, как обычно, в виде

$$
\operatorname{ad}(x)(v)=[x, v]
$$

инъективен, а значит, по соображениям размерностей, и биективен. Так как специальная ортогональная группа компактна и связна, то гомоморфизм Ad сюръективен. Ядро его состоит из таких элементов, которые коммутируют с векторами $v \in V$, то есть в точности из \pm 1 . Значит, это двулистное накрытие. Два элемента из ядра +1 и -1 могут быть связаны путем $\gamma:[0, \pi] \rightarrow \operatorname{Spin}(V)$, задаваемым формулой

$$
\gamma(t)=\cos (t)+\sin (t) \cdot e_{1} \cdot e_{2}
$$

Отсюда следует связность группы, и из того, что получается нетривиальное двулистное накрытие, следует, что $\operatorname{Spin}(V)$ компактна и односвязна. Последнее, кстати, также очевидно следует из того, что $\operatorname{Spin}(V)$ содержится в единичной сфере алгебры Клиф्ффорда.

Перейдем к рассмотрению комплексификации алгебр Клиффорда.

Обозначим через $C^{\mathbb{C}}(V)=C(V) \otimes \mathbb{C}$ такую комплексификацию. Базис $\left\{e_{I}\right\}$ так и останется базисом, а координаты станут комплексными. Инволюция в этом случае запишется в виде

$$
\bar{x}=\sum_{I} \varepsilon_{I} \cdot \overline{x_{I}} \cdot e_{I}
$$

как и вьше, $\overline{x \cdot y}=\bar{y} \cdot \bar{x}$, и имеется эрмитова форма

$$
\langle x, y\rangle=(\bar{x} \cdot y)_{0}=\sum_{I} \overline{x_{I}} \cdot y_{I}
$$

Так же, как и в вешественном случае, группа $\operatorname{Spin}^{\mathbb{C}}(V)$ определяется как множество элементов алгебры $x \in C^{+}(V) \otimes \mathbb{C}$ таких, что $\bar{x} \cdot x=1$ и $x \cdot V \cdot \bar{x}=V$. Алгебра Ли такой группы представляется в виде прямой суммы $C_{2}(V) \oplus i \mathbb{R}$, и група может быть записана в виде

$$
\operatorname{Spin}^{\mathbb{C}}(V)=\left\{e^{i \theta} \cdot x \mid \theta \in \mathbb{R}, x \in \operatorname{Spin}(V)\right\} .
$$


Следовательно, мы можем отождествить $\operatorname{Spin}^{\mathbb{C}}(V)=\operatorname{Spin}(V) \times_{\mathbb{Z}_{2}} S^{1}$, где действие групшы $\mathbb{Z}_{2}$, очевидно, диагональное. Такое отождествление дает точную последовательность

$$
1 \rightarrow S^{1} \rightarrow \operatorname{Spin}^{\mathbb{C}}(V) \rightarrow \mathrm{SO}(V) \rightarrow 1,
$$

где вторая стрелка - очевидное вложение окружности в $\operatorname{Spin}^{\mathbb{C}}(V)$, а третья - отображение $\mathrm{Ad}: \operatorname{Spin}^{\mathbb{C}}(V) \rightarrow \mathrm{SO}(V)$, строящееся так же, как и в вешественном случае.

Имеется и другая точная последовательность

$$
1 \rightarrow \operatorname{Spin}(V) \rightarrow \operatorname{Spin}^{\mathbb{C}}(V) \rightarrow S^{1} \rightarrow 1,
$$

в которой третья стрелка есть отображение $\delta: \operatorname{Spin}^{\mathbb{C}}(V) \rightarrow S^{1}$, задаваемое формулой $\delta\left(e^{i \theta} \cdot x\right)=e^{2 i \theta}$.

4.2. Спинорные расслоения. Если размерность $\operatorname{dim} V=2 n$ четна, тогда комплексификация алгебры Клиффорда представляется как алгебра эндоморфизмов $2^{n_{-}}$ мерного комплексного эрмитова пространства $W$. Более точно, такое отождествление есть изоморфизм алгебр $\Gamma: C^{\mathbb{C}}(V) \rightarrow \operatorname{End} W$, удовлетворяющий условиям

$$
\begin{aligned}
\Gamma(x+y) & =\Gamma(x)+\Gamma(y), \\
\Gamma(x \cdot y) & =\Gamma(x) \cdot \Gamma(y), \\
\Gamma(\bar{x}) & =\Gamma(x)^{*}
\end{aligned}
$$

для любых $x, y \in C^{\mathbb{C}}(V)$. В частности, если мы рассмотрим ограничение этого изоморфизма на естественно вложенное в алгебру само векторное пространство $V$, то для любого вектора $v \in V$ выполняются условия

$$
\begin{gathered}
\Gamma(v)^{*}+\Gamma(v)=0, \\
\Gamma(v)^{*} \cdot \Gamma(v)=|v|^{2} \cdot 1 .
\end{gathered}
$$

Свойства этого изоморфизма определяют его универсальность вместе с универсальностью самой алгебры. А именно, если $W^{\prime}$ - комплексное эрмитово пространство размерности $2^{n}$ и $\Gamma$ - линейное отображение $\Gamma: V \rightarrow$ End $W^{\prime}$, удовлетворяющее (4.18), тогда $Г$ естественно продолжается до изоморфизма $\Gamma: C^{\mathbb{C}}(V) \rightarrow \operatorname{End} W^{\prime}$, обладающего свойствами (4.17).

Приведем два примера.

Как мы видели в предыдушем разделе, алгебра Клиффордапространства $\mathbb{R}^{2}$ может быть отождествлена с кватернионами, при этом $i=e_{1}, j=e_{2}, k=e_{1} \cdot e_{2}$, а инволюция задается обычным сопряжением.

Тогда линейное отображение $\gamma: \mathbb{H} \rightarrow \mathbb{C}^{2 \times 2}$ задается формулой

$$
\gamma(x)=\left(\begin{array}{cc}
x_{0}+i x_{1} & x_{2}+i x_{3} \\
-x_{2}+i x_{3} & x_{0}-i x_{1}
\end{array}\right)
$$

Недолго проверить, что комплексификация отображения $\gamma^{\mathbb{C}}: \mathbb{H} \otimes_{\mathbb{R}} \mathbb{C} \rightarrow \mathbb{C}^{2 \times 2}$ является изоморфизмом алгебр, удовлетворяюшим (4.17).

Теперь рассмотрим отображение

$$
\Gamma: \mathbb{H} \rightarrow \mathbb{C}^{4 \times 4}
$$


задаваемое матрицей

$$
\Gamma(x)=\left(\begin{array}{cc}
0 & \gamma(x) \\
-\gamma(x)^{*} & 0
\end{array}\right)
$$

Такое отображение удовлетворяет условиям (4.18), поэтому может быть продолжено до изоморфизма $\Gamma: C^{\mathbb{C}}(\mathbb{H}) \rightarrow \mathbb{C}^{4 \times 4}$ алгебр.

Последняя формула показьвает, что в четырехмерном случае представление допускает разложение $W=\mathbb{C}^{2} \oplus \mathbb{C}^{2}$, сохраняющееся при действии четных элементов алгебры Клиффорда.

Выбрав базис, мы одновременно фиксируем ориентацию пространства $V$, и обозначим через

$$
\varepsilon=e_{2 n} \cdot \ldots \cdot e_{1} \in C(V)
$$

единичньй элемент степени $2 n$, получаемьй из положительно ориентированного базиса. Тогда $\varepsilon^{2}=(-1)^{n}$ и, следовательно, существует разложение $W=W^{+} \oplus W^{-}$, задаваемое условием

$$
W^{ \pm}=\left\{x \in W \mid \Gamma(\varepsilon) x= \pm i^{n} x\right\} .
$$

Заметим, что при смене ориентации пространства слагаемые $W^{+}$и $W^{-}$просто меняются местами. Несложно видеть, что $\Gamma(v)$ отображает $W^{+}{ }_{\text {в }} W^{-}$и наоборот. Поэтому четные элементы из $C^{+}(V) \otimes \mathbb{C}$ действуют с сохранением знака.

Далее, из всего сказанного вьше нетрудно видеть, что в случае размерности четыре сушествует гомоморфизм из $C^{0}(V) \otimes \mathbb{C} \oplus C^{2}(V) \otimes \mathbb{C} \oplus C^{4}(V) \otimes \mathbb{C} \cong \mathbb{C} \oplus C^{2}(V) \otimes \mathbb{C} \oplus \mathbb{C}$ в прямую сумму End $W^{+} \oplus$ End $W^{-}$. Элементы нулевой и четвертой степени имеют в качестве образа прямую сумму $\operatorname{diag}\left(W^{+}\right) \oplus \operatorname{diag}\left(W^{-}\right)$, и на долю средней компоненты вьпадает изоморфизм

$$
\Gamma: C^{2}(V) \otimes \mathbb{C} \rightarrow \operatorname{ad} W^{+} \oplus \operatorname{ad} W^{-},
$$

где $\operatorname{ad} W^{ \pm} \subset$ End $W^{ \pm}$- бесследовые эндоморфизмы.

Представление образа в виде прямой суммы поднимается до разложения $C^{2}(V) \otimes$ $\mathbb{C}$ в прямую сумму двух трехмерных слагаемых. Очевидно, это разложение задается действием элемента $\varepsilon$ - инволюцией на $C^{2}(V) \otimes \mathbb{C}$. Как всегда, по действию инволюции получаем два собственных подпространства - с собственньми значениями +1 и -1 . Несложно видеть, что первое из этих подпространств будет изоморфно $\mathrm{ad} W^{+}$, второе - ad $W^{-}$(это следует из согласованности умножения на $\varepsilon$ слева и справа в (4.22)).

Как видно из всего сказанного, мы постепенно переключились от общего описания Spin-представлений к конкретному частному случаю - когда размерность исходного пространства равна 4. Причина в том, что наша цель - именно этот случай. Заметим, что описание разложения $C^{2}(V) \otimes \mathbb{C}$ в прямую сумму по действию инволющии очень напоминает то, с чем мы встречались в $\S 2$ - разложение по действию оператора $*$. Эта аналогия приходит на ум не случайно, потому что данная конструкция в точности совпадает с $*$-разложением по модулю очевидного изоморфизма

$$
\Lambda^{2} V^{*} \rightarrow C_{2}(V): \eta=\sum_{i<j} \eta_{i, j} \cdot e_{i}^{*} \wedge e_{j}^{*} \mapsto \sum_{i<j} \eta_{i, j} \cdot e_{i} \cdot e_{j}
$$

Далее, в качестве композиции имеем гомоморфизм

$$
\begin{aligned}
& \rho: \Lambda^{+} \rightarrow \operatorname{ad} W^{+}, \\
& \rho: \Lambda^{-} \rightarrow \operatorname{ad} W^{-} .
\end{aligned}
$$


Теперь почти все готово к тому, чтобы описать конфигурационное пространство уравнения монополя.

Пусть $(X, g)$ - четырехмерное ориентируемое замкнутое многообразие с римановой метрикой. Рассмотрим комплексификацию его касательного пространства $T^{*} X \otimes \mathbb{C}$. В каждой точке $x \in X$ имеется комплексифицированная алгебра Клиффорда и, следовательно, пара комплексных эрмитовых пространств $W^{ \pm}$, причем вьполняются следуюшие соотношения:

$$
\begin{gathered}
T_{x} X \otimes \mathbb{C}=\operatorname{Hom}\left(W^{-}, W^{+}\right), \\
\Lambda^{2} T_{x}^{*} X=\Lambda^{+} \oplus \Lambda^{-}, \quad \operatorname{ad} W^{ \pm}=\Lambda^{ \pm} .
\end{gathered}
$$

Чтобы глобализовать эту конструкцию, необходимо напомнить некоторые определения.

$\mathrm{Spin}^{\mathbb{C}}{ }$-структурой на $n$-мерном ориентируемом расслоении $V \rightarrow X$ назьвается главное расслоение $P \rightarrow X$ со структурной группой $G=\operatorname{Spin}^{\mathbb{C}}(n)$ вместе с изоморфизмом

$$
P \times_{\operatorname{Spin}_{(n)} \mathbb{C}} \mathbb{R}^{n} \rightarrow V .
$$

Например, для $\mathrm{SO}(n)$-расслоения сушествование $\mathrm{Spin}^{\mathbb{C}}$-структуры означает, что имеется подъем соответствующего главного расслоения до главного расслоения со структурной групой $\operatorname{Spin}^{\mathbb{C}}(n)$. Такой подъем существует тогда и только тогда, когда второй класс Штиффеля-Уитни исходного расслоения $w_{2}(V) \in H^{2}\left(X, \mathbb{Z}_{2}\right)$ допускает целочисленный подъем. Этот подъем будет в точности равен первому классу Черна $c_{1}(L)$ линейного расслоения

$$
L=P \times_{\operatorname{Spin}^{\mathbb{C}}(n)} \mathbb{C},
$$

где действие групшы $\operatorname{Spin}^{\mathbb{C}}(n)$ на $\mathbb{C}$ определяется гомоморфизмом $\delta$ (см. (4.16)).

$\mathrm{Spin}^{\mathbb{C}}$-структурой на ориентируемом римановом многообразии $X$ называется такая структура на его касательном расслоении. Известно, что всякое компактное односвязное многообразие размерности 4 допускает $\operatorname{Spin}^{\mathbb{C}}$-структуру - так как существование такой структуры равносильно тому, что третий класс Штиффеля-Уитни многообразия равен нулю, а это следует из тривиальности третьих когомологий односвязного многообразия.

Очевидно, что $\mathrm{Spin}^{\mathbb{C}}$-структура задается с точностью до подкрутки на линейное $\mathbb{C}$-расслоение.

Можно определять Spin $^{\mathbb{C}}$-структуру на 4-многообразии просто заданием пары комплексных эрмитовых расслоений ранга два с одним и тем же дискриминантом, обладаюших свойствами (4.26). Заметим, что линейная подкрутка не нарушает этих свойств, поэтому выбором $\operatorname{Spin}{ }^{\mathbb{C}}$-структуры называется выбор класса $c \in H^{2}(X, \mathbb{Z})$, равного

$$
c=c_{1}\left(\operatorname{det} W^{ \pm}\right) .
$$

Мы будем назьвать $W^{+}$и $W^{-}$- комплексные эрмитовы расслоения ранга 2 - спинорными расслоениями правой и левой киральности соответственно, а элементы пространств их сечений - просто спинорами.

Заметим, что если многообразие $X$ допускает Spin-структуру, это означает, что в классах $\operatorname{Spin}^{\mathbb{C}}$-структур сушествует выделенная,

$$
c=c_{1}\left(\operatorname{det} W^{ \pm}\right)=0 .
$$


В келеровом случае Spin ${ }^{\mathbb{C}}$-структура существует всегда. Можно определить пару $\mathbb{C}^{2}$-расслоений $W^{ \pm}$явньми формулами. Пусть

$$
\begin{aligned}
& W^{+}=\bigoplus \Lambda^{*, \text { even }} T^{*} X, \\
& W^{-}=\bigoplus \Lambda^{*, \text { odd }^{*}} T^{*} X
\end{aligned}
$$

Разложение на $\Lambda^{+}$и $\Lambda^{-}$имеет в этом случае вид

$$
\begin{gathered}
\Lambda^{+} \otimes \mathbb{C}=\Lambda^{2,0} T^{*} X \oplus \mathbb{C} \cdot \omega \oplus \Lambda^{0,2} T^{*} X, \\
\Lambda^{1,1} T^{*} X \otimes \mathbb{C}=\mathbb{C} \cdot \omega \oplus \Lambda^{-} T^{*} X \otimes \mathbb{C},
\end{gathered}
$$

где $\omega$ - келерова форма.

Заметим, что существование Spin-структуры на келеровом многообразии равносильно четности канонического класса. Так как представление (4.31) дает $\operatorname{det} W^{ \pm}=$ $K^{-1}$, а подкрутка спинорных расслоений на линейное $E$ дает поправку детерминанта $\operatorname{det} W_{E}^{ \pm}=K^{-1} \otimes E^{2}$, последнее замечание очевидно.

Симплектическое многообразие также всегда имеет $\mathrm{Spin}^{\mathbb{C}}$-структуру. Рассмотрим некоторую почти комплексную структуру $I$, согласованную с кососимметрической формой $\omega$ (такие согласованные почти комплексные структуры образуют стягиваемое множество), и эта почти комплексная структура задаст на касательном расслоении структуру комплексного расслоения. Из невырожденности $I$ следует, что второй класс Черна этого расслоения будет равен нулю, а первый класс $c_{1}=c$ и будет $\operatorname{Spin}^{\mathbb{C}}$-структурой. Заметим, что из связности пространства почти комплексных структур, совместимых с кососимметрической формой $\omega$ (они образуют конус), и принадлежности класса $c$ пространству целочисленных когомологий следует независимость $\mathrm{Spin}^{\mathbb{C}}$-структуры от выбора почти комплексной структуры.

Заметим, что как только на касательном расслоении симплектического многообразия выбрана почти комплексная структура, то сразу же этим выбором автоматически задается и метрика (а все вместе они образуют эрмитову тройку $(\omega, I, g))$, которая доставляет спинорное разложение (см. 33$)$, совпадающее с тем, которое доставляет та же почти комплексная структура. Значит, в симплектическом случае спинорное разложение не зависит от метрики, как не зависит оно от почти комплексной структуры.

4.3. Уравнение А-монополя. Как было замечено во введении, мы не можем называть решения описьваемой ниже системы уравнений абелевыми монополями, хотя математическая традиция это и допускает. Мы будем называть их А-монополями, чтобы отличить их от В-монополей следующего параграфа.

Пусть $(X, g)$ - гладкое, компактное, односвязное риманово многообразие. Существует единственная связность на касательном расслоении, совместимая с метрикой и не имеющая кручения, - это связность Леви-Чивита. Выбор Spin ${ }^{\mathbb{C}}$-структуры дает нам пару комплексных эрмитовых расслоений ранга 2 с выбранным нами классом $c \in H^{2}(X, \mathbb{Z})$ таким, что $c=c_{1}\left(\operatorname{det} W^{ \pm}\right)$.

Посредством гомоморфизма $\rho$ связность Леви-Чивита индуцирует связности на $\operatorname{ad} W^{ \pm}$, определяющие связности на расслоениях с точностью до задания связности $a$ на линейном расслоении $L=\operatorname{det} W^{+}=\operatorname{det} W^{-}$. Заданием такой связности мы определяем ковариантное дифференцирование на пространстве сечений $\Gamma\left(W^{ \pm}\right)$. 
Конфигурационным пространством уравнения монополя будет множество пар $(a, \Phi)$, где первьй элемент - унитарная связность на $L$, а второй - сечение спинорного расслоения правой киральности, профакторизованное по действию группы $\mathscr{G}=$ Aut $L=\operatorname{Map}\left(X, S^{1}\right)$. Мы будем назьвать эту пару парой (связность + спинор). Таким образом, $a \in \mathscr{A}(L), \Phi \in \Gamma\left(W^{+}\right)$, и пара как элемент конфигурационного пространства $(a, \Phi) \in\left(\mathscr{A}(L) \times \Gamma\left(W^{+}\right)\right) / \mathscr{G}$.

Введем оператор Дирака $D_{a}-$ дифференциальный оператор на пространствах сечений спинорных расслоений, задаваемьй следующим образом. Ковариантная производная задает отображение

$$
d_{a}: \Gamma\left(W^{+}\right) \rightarrow \Gamma\left(W^{+}\right) \otimes \Gamma\left(T^{*} X\right),
$$

и последнее тензорное произведение выражается как

$$
\Gamma\left(W^{+} \otimes T^{*} X\right)=\Gamma\left(W^{+} \otimes \operatorname{Hom}\left(W^{-}, W^{+}\right)^{*}\right)=\Gamma\left(W^{-}\right)
$$

посредством клиффордова умножения. Такая композищия - ковариантного дифференширования и свертки - и назьвается оператором Дирака

$$
D_{a}: \Gamma\left(W^{+}\right) \rightarrow \Gamma\left(W^{-}\right)
$$

Сразу же введем формально сопряженный оператор $D_{a}^{*}$, задаваемьй равенством

$$
\int_{X}\left(D_{a}^{*}(\Phi), \Psi\right) d \mu=\int_{X}\left(\Phi, D_{a}(\Psi)\right) d \mu
$$

для любых $\Phi \in \Gamma\left(W^{-}\right), \Psi \in \Gamma\left(W^{+}\right)$. Таким образом, сопряженньй оператор действует в обратную сторону

$$
D_{a}^{*}: \Gamma\left(W^{-}\right) \rightarrow \Gamma\left(W^{+}\right) .
$$

Уравнение монополя, естественно, должно быть калибровочно инвариантно. Для оператора Дирака таким может быть только $D_{a}(\Phi)=0$.

Вторым уравнением будет $\rho\left(F_{a}^{+}\right)=i \cdot(\Phi \otimes \bar{\Phi})_{0}$. Черта в правом выражении означает эрмитово сопряжение, а умножение на мнимую единицу соответствует тому, что $F_{a}^{+}-$мнимозначная форма. В дальнейшем для краткости мы будем опускать значок $\rho($ ), так как это не может привести к недоразумению.

Проверка инвариантности второго уравнения также очевидна - из-за простоты абелевой калибровочной группы, действующей на слоях спинорных расслоений умножением на комплексные числа.

Итак, уравнение монополя для пар $(a, \Phi)$ имеет вид

$$
\begin{gathered}
D_{a}(\Phi)=0, \\
F_{a}^{+}=i \cdot(\Phi \otimes \bar{\Phi})_{0} .
\end{gathered}
$$

Многообразие модулей монополей $\mathscr{M}_{c}$ есть множество орбит решений уравнения монополя по действию калибровочной групшы $\mathscr{G}=$ Aut $L$.

В качестве первого шага найдем виртуальную размерность многообразия модулей монополей. Для этого рассмотрим эллиптический комплекс, отвечающий линеаризации уравнения монополя. Но запишем сначала саму линеаризацию. 
Линеаризация задается малой вариацией $(a+\delta a, \Phi+\delta \Phi)$, где, очевидно, $\delta a \in$ $\Omega^{1}($ End $L)=\Omega^{1}$, а спинорная вариация $\delta \Phi \in \Gamma\left(W^{+}\right)$. Тогда линеаризованное уравнение вьглядит так:

$$
\begin{gathered}
D_{a}(\delta \Phi)=-\delta a * \Phi, \\
d_{a}^{+}(\delta a)=i \cdot(\Phi \otimes \overline{\delta \Phi}+\delta \Phi \otimes \bar{\Phi}) .
\end{gathered}
$$

Звездочка в первом уравнении линеаризации означает спинорное умножение.

Поэтому эллиптический комплекс, соответствующий линеаризации, имеет вид

$$
0 \longrightarrow \Omega^{0} \stackrel{s}{\longrightarrow} \Omega^{1} \oplus \Gamma\left(W^{+}\right) \stackrel{t}{\longrightarrow} \Omega^{+} \oplus \Gamma\left(W^{-}\right) \longrightarrow 0 .
$$

Здесь отображение $t$ отвечает линеаризации уравнения монополя, а $s$-отображение из 0-форм в малую деформацию пары $(a, \Phi)$, отвечающую инфинитезимальному действию калибровочной группы.

Составим оператор $T=s^{*} \oplus t$, действующий на прямой сумме:

$$
T: \Omega^{1} \oplus \Gamma\left(W^{+}\right) \rightarrow \Omega^{0} \oplus \Omega^{+} \oplus \Gamma\left(W^{-}\right) .
$$

Виртуальная размерность многообразия модулей $\mathscr{M}_{c}$ равна индексу оператора $T$ (мы рассматриваем все расслоения как вешественные, т.е. размерности комплексных расслоений удваиваются). Забывая про члены нулевого порядка, входяшие в $T$, получаем, что индекс его равен сумме индексов оператора $d+d^{*}$ и оператора Дирака. Это следует из возможности деформации оператора $T$ в прямую сумму последних. Если $\chi$ и $\sigma$-эйлерова характеристика и сигнатура многообразия $X$, то индекс первого оператора равен $-\frac{1}{2}(\chi+\sigma)$, а индекс оператора Дирака (вешественньй) равен $\frac{1}{4}\left(c_{1}(L)^{2}-\sigma\right)$. Вместе получается

$$
d(L)=\frac{1}{4}\left(c_{1}(L)^{2}-(2 \chi+3 \sigma)\right)
$$

Если $d(L)<0$, то для общей метрики решений нет. В случае нулевой размерности, когда $d(L)=0$, многообразие модулей монополей для общей метрики состоит из конечного числа точек. Каждой точке можно присвоить знак, в зависимости от знака детерминанта $T$, и просуммировать по всем точкам. Полученное число обозначим $N(L, g)$. Пусть, как обьчно в наших рассмотрениях, $b^{+}(X)>1$. Тогда не только для обшей метрики $g_{0}$ не сушествует неприводимых решений, но любые две обшие метрики $g_{0}, g_{1}$ могут быть соединены путем в пространстве метрик $\gamma:[0,1] \rightarrow \mathscr{H}$, $\gamma(0)=g_{0}, \gamma(1)=g_{1}$, таким, что для любого $t$ метрика $g(t)$ не допускает приводимых решений. Тогда функция $N(L, t)=N(L, g(t))$ должна быть константой, будучи непрерьвной целочисленной функцией. Из этого следует, что число $N(L, g)$ на самом деле не зависит от выбора общей метрики, а значит, оно является инвариантом гладкой структуры. Выбор Spin ${ }^{\mathbb{C}}$-структуры, необходимый для определения уравнения монополя, при отсутствии 2 -кручения в $H^{2}(X, \mathbb{Z})$ эквивалентен выбору класса $c=c_{1}(L) \in H^{2}(X, \mathbb{Z})$. Отсюда определяется целочисленная функция $N_{S W}(c) \in \mathbb{Z}$, которая инвариантна относительно действия группы диффеоморфизмов, сохраняющих ориентацию.

Уравнения инстантона и монополя оказались чрезвычайно полезньми для математиков. Поэтому для них естественен вопрос, а как вообще ишутся "полезные” уравнения и не продолжить ли им поиск таковых? Напомним вкратце историю появления уравнений такого сорта в физике. 
Прежде всего, такие уравнения минимизируют лагранжианы классических полевых теорий. Яркий пример: пусть $(X, g, E)$ - обычная тройка геометрических объектов, где $X$ - многообразие, $g$ - метрика на нем и $E-\mathrm{U}(n)$-векторное расслоение. Конфигурационное пространство классической системы - пространство пар

$$
(a, \phi) \in \mathscr{A}_{h} \times \Gamma(E),
$$

где $a$ - унитарная связность на $E$, а $\phi$ - сечение расслоения $E$ (ситуация, когда говорят, что имеется $n$ цветов и один аромат). Функционал Янга-Миллса-Хиггса имеет вид

$$
\mathrm{YMH}_{\tau}(a, \phi)=\left\|F_{a}\right\|^{2}+\left\|d_{a}(\phi)\right\|^{2}+\frac{1}{4}\left\||\phi|_{h}^{2}-\tau\right\|^{2}
$$

Если $X$ двумерно, то конформньй класс метрики задает комплексную структуру на $X$, так что ковариантная производная раскладьвается в прямую сумму по битипам $d_{a}=d_{a}^{\prime}+d_{a}^{\prime \prime}$. Классические решения (минимумы $\mathrm{YMH}_{\tau}$ ) удовлетворяют уравнениям

$$
d_{a}^{\prime \prime}(\phi)=0, \quad \Lambda_{\omega} \cdot F_{a}-\frac{1}{2} i|\phi|_{h}^{2}+\frac{1}{2} i \tau=0
$$

где $\Lambda_{\omega}$ - оператор, формально сопряженньй внешнему умножению на келерову форму $\omega$. Это знаменитое уравнение вихрей (инвариантное относительно действия калибровочной группы), которое Ландау и Гинзбург в 1951 году использовали для описания сверхпроводимости: $X=S^{2}, E=L$ - линейное расслоение, сечение фголоморфно и его орбита однозначно определяется конечным множеством его нулей (каждьй из которых интерпретировался как “вихрь”). Сверхпроводимость при этом соответствует конденсации вихрей.

Уравнение вихрей (4.44) и подобные ему можно решать для любой келеровой метрики на многообразии любой размерности. Как обобщить уравнение вихря на случай любой метрики (а не только согласованной с некоторой комплексной структурой)? Ответ вполне логичен: $d_{a}^{\prime \prime}$ нужно заменить на оператор Дирака, а $\phi-$ на спинорное поле. Тогда орбита решения назьвается "монополем". Это название - счастливое, потому что $S$-двойственность (см. [18], [12]) тогда формулируется в точности так же, как это делал Дирак для электромагнитных полей. Лагранжиан теперь пишется в виде

$$
S W(a, \phi)=\frac{1}{2}\left\|F_{a}\right\|^{2}+\left\|d_{a}(\phi)\right\|^{2}+\frac{1}{8}\left\||\phi|_{h}^{2}-R\right\|^{2},
$$

где $R$ - скалярная кривизна метрики.

Замечательно, что почти через 40 лет Зайберг и Виттен дали описание механизма трех фаз квантового вакуума (в суперсимметричной теории Янга-Миллса): конденсации, конфайнмента и фазы Хиггса, абсолютно в тех же терминах, что и Ландау и Гинзбург.

Последний вопрос: почему используется столь "бедная" конфигурация полей? A priori мы могли бы добавить гораздо больше полей Хиггса (ароматов), чтобы получить более богатую конфигурацию. Но в 50-х годах физики обнаружили, что для того чтобы можно было вычислять квантовые корреляционные функции в квазиклассическом пределе, нужно, чтобы бета-функция Гелмана-Лоу полей этой теории была отрицательной. То есть для успешного квантования нужно наложить 
чрезвычайно стеснительные ограничения на конфигурацию полей. Необходимость забраковать большинство теорий была названа эффектом “московского нуля", и Ландау даже предложил устроить торжественные похороны лагранжиана. Однако “бедность не порок”, и даже бедные конфигурации приводят к блестящему развитию теории (см., например, [25]). А для полного описания конфигураций для $\mathbb{C P}^{2}$ и связи с исключительными расслоениями см. [12]. Будут ли полезны "не квантуюшиеся" классические теории для математиков - вопрос открытый, но при их исследовании математики не могут рассчитьвать на помощь физиков.

4.4. Инварианты Зайберга-Виттена. Итак, как уже было сказано вьше, Зайберг и Виттен рассматривают случай, когда многообразие модулей монополей имеет нулевую размерность, т.е. состоит просто из точек. Чтобы составить альтернированную сумму, необходимо убедиться в конечности множества решений уравнения монополя (в этом разделе мы будем иметь дело только с А-монополями и будем сокрашать обозначения) в этом случае.

Конечность множества решений следует из компактности $\mathscr{M}_{c}$. Для доказательства компактности необходимо воспользоваться формулой Вейщенбока

$$
D_{a}^{*} D_{a}(\Phi)=\nabla_{a}^{*} \nabla_{a} \Phi+\frac{1}{4} R \Phi-\frac{1}{2} F_{a}^{+}(\Phi)
$$

где $\Phi$ - произвольньй спинор правой киральности, а $R$ - скалярная кривизна многообразия $X$. Тогда для решения уравнения монополя справедливо утверждение

$$
|\Phi|^{2} \leqslant \max (0,-R),
$$

причем квадрат модуля спинора берется в той точке многообразия, где он максимален.

Вькладки достаточно просты: в точке, где $|\Phi|^{2}$ максимален, вьгисляем

$$
\begin{aligned}
0 & \leqslant \Delta|\Phi|^{2}=2\left\langle\nabla_{a}^{*} \nabla_{a} \Phi, \Phi\right\rangle+2\left\langle\nabla_{a} \Phi, \nabla_{a} \Phi\right\rangle \leqslant 2\left\langle\nabla_{a}^{*} \nabla_{a} \Phi, \Phi\right\rangle \\
& =-\frac{1}{4} S|\Phi|^{2}+\left\langle i(\Phi \otimes \bar{\Phi})_{0} \Phi, \Phi\right\rangle=-\frac{1}{2} S|\Phi|^{2}-\frac{1}{2}|\Phi|^{4} .
\end{aligned}
$$

И если в точке своего максимума $|\Phi|^{2}$ отличен от нуля, то после очевидного деления получаем условие (4.47).

Это условие дает общую $C^{0}$-ограниченность спинорной части любого решения, из чего путем стандартного рассуждения получается компактность многообразия модулей монополей. Кроме того, это условие показьвает, что нетривиальных решений уравнения монополя нет на многообразиях, допускающих положительную скалярную кривизну. А тривиальных нет, потому что нет абелевых инстантонов-в случае общей метрики.

Некомпактность многообразия модулей инстантонов возникала из-за того, что предельными связностями выступали идеальные связности (см. раздел 3.1 настоящего обзора). Они, в свою очередь, возникали изначально на $\mathbb{R}^{4}$, как результаты сжатия инстантонов. Но так как $\mathbb{R}^{4}$ - конформно плоское пространство, скалярная кривизна которого равна нулю, то монополей на этом пространстве не сушествует. И поэтому не существует "сжатых" монополей, могущих нарушать компактность $\mathscr{M}_{c}$.

Вернемся к определению инвариантов. Как уже было указано, в случае равенства нулю индекса оператора $T=T(c)$ многообразие модулей монополей нульмерно, т.е. 
состоит из некоторого множества точек конфигурационного пространства, причем конечного множества - это следует из компактности $\mathscr{M}_{c}$. Каждой такой точке можно сопоставить знак $\varepsilon_{i, c}= \pm 1$ в зависимости от знака детерминанта $T$. Составим альтернированную сумму:

$$
N(c)=\sum_{i} \varepsilon_{i, c}
$$

которая при естественных условиях трансверсальности и будет инвариантом подлежащего многообразия.

Для того чтобы выяснить, определен ли знак детерминанта $T$, достаточно опять деформировать этот оператор в прямую сумму операторов Дирака и $d+d^{*}$. Первьй из них имеет естественную ориентацию - как комплексньй оператор, рассматриваемьй как вешественный. Второй не зависит от связности и спинора (т.е. от точки конфигурационного пространства), и его детерминантное расслоение тривиализуется раз и навсег да выбором ориентации на $H^{1}(X, \mathbb{R}) \oplus H^{+}(X, \mathbb{R})$. Заметим, что такой же выбор был необходим для ориентации и в теории Дональдсона.

Вообще, число решений системы уравнений с учетом знака детерминанта оператора, похожего на $T$, является топологическим инвариантом, если может быть установлена подходящая компактность, т.е., в нашем случае, если калибровочно инвариантная система уравнений имеет решения, обладаюшие компактностью и свободой действия на них калибровочной групш. О компактности уже было сказано выше, а свобода следует из того, что нет тривиальных решений, так как нет абелевых инстантонов.

Действительно, абелевых инстантонов в случае неопределенной формы и общей метрики нет по следующей причине. Так как $F /(2 \pi)$ представляет первый класс Черна расслоения $L$ в когомологиях, то это целочисленный класс. Если $F^{+}=0$, то $F /(2 \pi)$ лежит в пересечении целочисленной решетки в $H^{2}(X, \mathbb{R})$ и подпространства антиавтодуальных гармонических классов $H^{-}(X, \mathbb{R})$. Это подпространство не пусто, так как мы предполагаем $b_{2}^{+} \geqslant 1$. При изменении метрики это подпространство врашается вокруг нуля, и в случае общей метрики такое пересечение состоит только из нуля (теория вещественных периодов римановых метрик).

Следовательно, для общей метрики абелевых инстантонов нет и инвариант $n_{c}$ вполне определен. Исключение составляет случай, когда $c=0$ и сушествует тривиальньй абелев инстантон. Для определения инварианта $n_{0}$ необходимо деформировать уравнение монополя, возмутив с помощью автодуальной формы уравнение на кривизну

$$
F_{a}^{+}=i(\Phi \otimes \bar{\Phi})_{0}-\eta
$$

Этим возмущением мы достигаем свободы действия калибровочной групшы на пространстве решений, а дальше действуем как и прежде - находим число точек, считаем альтернированную сумму и получаем $n_{0}$. Это число не будет зависеть от непрерьвной деформации формы $\eta \neq 0$, и если предположить $b_{2}^{+}>1$, то из-за связности пространства всех возможных $\eta$ получаем целочисленный топологический инвариант. Как уже обсуждалось в разделе 4.3, это дает инвариант гладкой структуры подлежащего многообразия.

Необходимо отметить, что инварианты $N(c)$ не равны нулю только для достаточного малого подмножества классов когомологий, которые принято назьвать базисными 
классами теории Зайберга-Виттена. Множество этих классов (очевидно инвариантное относительно смены знака)

$$
\left\{ \pm \beta_{1}, \ldots, \pm \beta_{N}\right\}_{S W} \subset H_{\mathrm{spin}}^{2}(X, \mathbb{Z})
$$

во всем множестве Spin ${ }^{\mathbb{C}}$-структур (опять же по модулю тривиальности 2-кручения) определяет полином Лорана

$$
S W_{X}=n_{0}+\sum_{i=1}^{N} n\left(\beta_{i}\right)\left(t_{i}+(-1)^{\frac{b^{+}+1}{2}} t_{i}^{-1}\right),
$$

где, в точности как в (3.38), переменная $t_{i}=\exp \left(\beta_{i}\right)$ с формальным правилом $t_{\alpha+\beta}=$ $t_{\alpha} t_{\beta}$.

Подрешетка $L_{S W} \subset H^{2}(X, \mathbb{Z})$, натянутая на базисные классы, инвариантна относительно групшы $\operatorname{Diff}^{+}(X)$. Это - аналог решетки Пикара алгебраической поверхности (по меньшей мере - по своей важности). Однако нетривиальность ее мы можем гарантировать также для келеровых и симплектических многообразий (и “близких" к ним - см. $\S 6)$.

Приведем краткую схему отыскания базисных классов в этих случаях. Заметим, что условие интегрируемости почти комплексной структуры нигде не требуется, поэтому случаи почти не различаются: наличие почти комплексной структуры $I$ равносильно сушествованию пары спинорного расслоения $W^{+}$с $c_{2}\left(W^{+}\right)=0$, более того, допускаюшего разложение $W^{+}=\mathscr{O} \oplus K^{-1}$. То есть спинорное расслоение с точностью до линейной подкрутки - выбора $\mathrm{Spin}^{\mathbb{C}}$-структуры - раскладьвается в прямую сумму

$$
W^{+} \otimes L=L \oplus\left(K^{-1} \otimes L\right),
$$

где $K$ - каноническое расслоение.

Обозначим компоненты разложения спинора $\Phi$ в первом слагаемом $\alpha$, а во втором $-i \bar{\beta}$. Второе уравнение монополя раскладьвается в систему

$$
\begin{aligned}
& F^{2,0}=\alpha \beta, \\
& F_{\omega}^{1,1}=-\frac{1}{2} \omega\left(|\alpha|^{2}-|\beta|^{2}\right), \\
& F^{0,2}=\bar{\alpha} \bar{\beta},
\end{aligned}
$$

где $\omega$ - келерова форма. Из соображений симметрии функционала действия в этом случае следует, что если рассмотреть операцию

$$
a \mapsto a, \quad \alpha \mapsto \alpha, \quad \beta \mapsto-\beta,
$$

то решения уравнения переходят в решения. Из этого следует, что для такого рода решений

$$
0=F^{2,0}=F^{0,2}=\alpha \beta .
$$

Равенство нулю $F^{0,2}$ в точности означает, что связность $a$ задает на $L$ голоморфную структуру. И базисные классы (определяемые условием, что (4.54) имеет решения) имеют ходжев тип $(1,1)$ для любой келеровой структуры на $X$. 
Способ различить, какое из сечений $-\alpha$ или $\beta$ - обращается в нуль, достаточно прост: проинтегрируем $(1,1)$-часть уравнений $(4.54)$ и получим

$$
\frac{1}{2 \pi} \int_{X} \omega \wedge F=-\frac{1}{4 \pi} \int_{X} \omega \wedge \omega \cdot\left(|\alpha|^{2}-|\beta|^{2}\right) .
$$

Слева стоит топологический инвариант, а именно $J=[\omega] c_{1}(L)$, равенство которого нулю означает сушествование нетривиальных абелевых инстантонов. По нашему соглашению мы рассматриваем только те метрики, для которых таковых нет. Тогда если $J<0$, то $\alpha \neq 0, \beta=0$, и наоборот.

Если $J<0$, то многообразие модулей монополей естественно отождествить с парами, состояшими из комплексной структуры на $L$ и ненулевого голоморфного сечения расслоения $L$, с точностью до умножения. В случае $J>0$ вместо этого расслоения надо рассмотреть $K^{-1} \otimes L$.

В нашем односвязном случае комплексная структура на $L$ (если она существует) единственна и многообразие модулей решений есть просто комплексное проективное пространство $\mathbb{P} H^{0}\left(X, K^{-1} \otimes L\right)$ или $\mathbb{P} H^{0}(X, L)$, в зависимости от знака $J$.

Заметим, что эти проективные пространства часто имеют размерность $\frac{b^{+}+1}{2}$, большую, чем виртуальная размерность многообразия модулей монополей. Это следствие того, что келерова метрика далеко не общая, а келерова геометрия не “трансверсальна" (см. [23]).

Чтобы преодолеть этот эффект, необходимо возмутить уравнение монополя, как это было сделано в (4.50). Для келерова многообразия условие $b_{2}^{+}>1$ эквивалентно тому, что $H^{2,0} \neq 0$, поэтому мы можем выбрать возмушение в виде ненулевой голоморфной 2-формы $\eta$. Тогда возмущенное уравнение монополя раскладьвается в систему

$$
\begin{aligned}
& F^{2,0}=\alpha \beta-\eta, \\
& F_{\omega}^{1,1}=-\omega\left(|\alpha|^{2}-|\beta|^{2}\right), \\
& F^{0,2}=\bar{\alpha} \bar{\beta}-\bar{\eta},
\end{aligned}
$$

а часть с оператором Дирака остается той же, что и вьше. При этом естественно ограничиться случаем, когда первьй класс Черна $L$ имеет тип $(1,1)$, иначе невозмущенное многообразие модулей пусто.

Как и ранее, преобразование

$$
a \mapsto a, \quad \alpha \mapsto \alpha, \quad \beta \mapsto \beta, \quad \eta \mapsto-\eta
$$

не является автоморфизмом уравнения монополя, но оставляет неизменным функционал действия, значит, решения под действием этого преобразования переходят в решения. Поэтому

$$
0=F^{0,2}=F^{2,0}=\alpha \beta-\eta,
$$

и опять условие $0=F^{0,2}$ означает, что связность определяет на $L$ голоморфную структуру.

И теперь мы можем видоизменить определение базисных классов:

1) это классы вида $x=-c_{1}(L)$, которые

2) для любого выбора $\eta \in H^{0}(X, K)$ допускают разложение $\eta=\alpha \cdot \beta$, где $\alpha, \beta-$ сечения расслоений $L$ и $K^{-1} \otimes L$ соответственно, причем

3) $x^{2}=K^{2}$. 
Для более явного описания определим класс $\eta$ как сумму неприводимых компонент $C_{i}$ с кратностями $r_{i}$. Тогда

$$
c_{1}(K)=\sum_{i} r_{i}\left[C_{i}\right]
$$

и сушествование разложения $\eta=\alpha \beta$ означает, что

$$
c_{1}(L)=\sum_{i} s_{i}\left[C_{i}\right]
$$

где кратности $s_{i}$ удовлетворяют неравенствам $0 \leqslant s_{i} \leqslant r_{i}$. Первый класс Черна расслоения $L$, следовательно, равен

$$
c_{1}(L)=\sum_{i}\left(s_{i}-\frac{1}{2} r_{i}\right)\left[C_{i}\right],
$$

и базисные классы по условию 1) имеют вид

$$
x=-\sum_{i}\left(s_{i}-r_{i}\right)\left[C_{i}\right]
$$

Последнее условие $\left(x^{2}=K^{2}\right)$ нуждается в рассмотрении в каждом конкретном случае. Например, для эллиптической поверхности $C_{i} C_{j}=0$ для любых $i$ и $j$, поэтому это условие выполняется автоматически:

$$
x^{2}=K^{2}=0 .
$$

Теперь вернемся к вопросу о знаке вклада точечного решения уравнения монополя, т.е. к вопросу об ориентации.

Рассмотрим обратимое линейное отображение $T: E \rightarrow F$ двух вешественных равноразмерных четномерных пространств, снабженных комплексными структурами. Если $T$ сохраняет комплексную структуру, то $\operatorname{det} T>0$, а если обращает, тогда знак детерминанта зависит от четности комплексной размерности $E$. А именно, $\operatorname{sign}(\operatorname{det} T)=$ $(-1)^{w}$, где $w=\operatorname{dim}_{\mathbb{C}} E$. Пусть $T$ сохраняет комплексную структуру, но не является обратимьм, а оператор $T^{\prime}: E \rightarrow F$, взаимно однозначно отображающий $\operatorname{ker} T$ на coker $T$, будет обрашающим ориентацию. Тогда для малого вещественного $\varepsilon$ знак $\operatorname{det}\left(T \oplus \varepsilon T^{\prime}\right)$ совпадает с $(-1)^{w}$, где, как и вьше, $w=\operatorname{dim}_{\mathbb{C}} \operatorname{ker} T$.

Расширим эти рассуждения на случай двух векторных расслоений $U$ и $V$, эллиптического оператора $T$, действующего на сечениях $\Gamma(U) \rightarrow \Gamma(V)$, нулевого индекса и достаточно тонкой деформации $T^{\prime}$. В этом случае $\operatorname{det}\left(T \oplus \varepsilon T^{\prime}\right)$ понимается как детерминант Рея-Зингера-Квиллена.

В нашем случае $T$ - линеаризация невозмушенного уравнения монополя, а $T^{\prime}$ - коррекция, пропорциональная $\eta$.

Как в (4.40), оператор $T$ действует на сечениях расслоения $U=\Omega^{1} \oplus W^{+}$, причем второе слагаемое рассматривается как вешественное векторное расслоение с естественной комплексной структурой. Тогда и на первом нетрудно задать комплексную структуру, действующую на $\Omega^{1,0}$ и $\Omega^{0,1}$ умножением на $+i$ и $-i$ соответственно. Похожим образом и на $V=\Omega^{0} \oplus \Omega^{+} \oplus W^{-}$задается комплексная структура, совпадающая с естественной на третьем слагаемом, действующая на $\Omega^{0,2}$ и $\Omega^{2,0}$ опять же 
умножением на $+i$ и $-i$ соответственно и меняющая местами остальные два слагаемые. Несложно видеть, что $T$ сохраняет ориентацию, а $T^{\prime}$ обращает.

Пусть $J>0$, тогда $\beta=0$ при $\eta=0$ и вклад решения, соответствующего разложению $\eta=\alpha \cdot \beta$, равен $(-1)^{w}$, где $w=\operatorname{dim}_{\mathbb{C}} \operatorname{ker} T$. Опишем этот вклад алгебро-геометрически: рассмотрим точную последовательность пучков

$$
0 \longrightarrow \mathscr{O} \stackrel{\alpha}{\longrightarrow} L \longrightarrow \mathscr{O}_{C}(D) \longrightarrow 0,
$$

где $L=\mathscr{O}(D)$, а $C$ - кривая нулей вложения $\alpha$. Ядро оператора $T$ имеет ту же размерность, что и $H^{0}\left(X, \mathscr{O}_{C}(D)\right)$, поэтому вклад в точности совпадает с размерностью полного линейного ряда соответствуюшего дивизора $|D|$ (или $D-K$ в случае $J<0$ ).

Прямым следствием этого (и формул (4.60)-(4.63)) является следующее утверждение.

ПРЕДЛОЖЕНИЕ 4.1. Пусть $X$ - односвязная алгебраическая поверхность, каноническая система которой содержит неприводимый әффективный дивизор D. Тогда

(i)

$$
S W_{X}=t+(-1)^{p_{g}(X)} t^{-1},
$$

где $t=\exp ([D])$, a $p_{g}-$ геометрический род поверхности;

(ii) для К3-поверхности

$$
S W_{\mathrm{K} 3}=1
$$

(iii) для поверхности $E(n)$ с әллиптическим пучком с сечением геометрического рода $n-1$

$$
S W_{E(n)}=\left(t+(-1)^{n-1} t^{-1}\right)^{n-2} .
$$

Такие же рассуждения можно провести и в других частных случаях алгебраических поверхностей. Важньй вьвод:

ПРЕДЛОЖЕНИЕ 4.2. ДЛя алгебраической поверхности положительного геометрического рода

(i) канонический класс всегда является фундаментальным классом ( частности $\left.S W_{X} \neq 0\right)$;

(ii) коэффициент (4.52) $n_{K}= \pm 1$;

(iii) полином Лорана является унимодулярным по $t_{K}$, т.е. его старший коәффичиент равен \pm 1 .

Эти утверждения справедливы и в большей общности.

ПРЕДЛОЖЕНИЕ 4.3 (Таубс). Утверждения (i)-(iii) предложения 4.2 верны и для симплектического многообразия с $b^{+}>1$.

Совсем немного нужно прибавить к нашим аргументам и конструкциям (4.54)-(4.56) и (4.59), чтобы получить полное доказательство этого факта (см., например, [17]).

С самого начала чувствовалась связь инвариантов Дональдсона с инвариантами Зайберга-Виттена. В частности, с помощью новых инвариантов можно доказать те же утверждения, что были доказаны Дональдсоном. 
Например, факт о том, что инварианты связной суммы двух многообразий с положительными $b_{2}^{+}$равны нулю, показьвает и Виттен, но уже для инвариантов, возникающих из уравнения монополя.

Прямая связь инстантонов и монополей представлена бордизмом, доставляемьм неабелевыми монополями (В-монополями), речь о которых пойдет в следующем параграф̆е.

\section{$\S$ 5. Неабелевы монополи}

В этом параграфе мы определим неабелевы монополи (В-монополи). При определении конфигурационного пространства на сей раз будет полезно геометрическое описание $\operatorname{Spin}^{\mathbb{C}}$-структур для четырехмерных многообразий.

5.1. Конфигурационное пространство. Рассмотрим гладкое компактное риманово 4-многообразие $(X, g)$ и $E-\mathrm{U}(2)$-расслоение на $M$, т.е. $\mathbb{C}^{2}$-расслоение с фиксированной эрмитовой формой $h$ и классами Черна $c_{1}, c_{2}$. Пусть $\mathscr{A}(E)-$ аффиннное пространство эрмитовых связностей на $E$ и $\mathscr{G}_{E}$ - группа калибровочных преобразований $E_{h}$, т.е. группа унитарных автоморфизмов $E$. Тогда разложение в ортогональную прямую сумму алгебр Ли определяет точную последовательность

$$
1 \rightarrow \mathscr{G}_{\Lambda^{2} E} \rightarrow \mathscr{G}_{E} \rightarrow \mathscr{G}_{\mathrm{ad} E} \rightarrow 1
$$

где $\Lambda^{2} E$ - линейное расслоение с индуцированной эрмитовой формой. Поэтому мы имеем изоморфизмы аффинных пространств

$$
\mathscr{A}(E)=\mathscr{A}(\operatorname{ad} E)
$$

и пространств классов связностей по модулю действия калибровочной группы:

$$
\mathscr{B}(E)=\mathscr{A}(E) / \mathscr{G}(E), \quad \mathscr{B}(\operatorname{ad} E)=\mathscr{A}(\operatorname{ad} E) / \mathscr{G}(\operatorname{ad} E), \quad \mathscr{B}\left(\Lambda^{2} E\right)=\mathscr{A}\left(\Lambda^{2} E\right) / \mathscr{G}\left(\Lambda^{2} E\right),
$$

которые преврашаются в банаховы орбифолды после выбора индексов соболевских норм на пространствах и группах (см., например, гл. 1 в [15]). Те же пространства, но без приводимых связностей, дают главное $\mathscr{G}$-расслоение

$$
\mathscr{A}(\operatorname{ad} E)^{*} \rightarrow \mathscr{B}(\operatorname{ad} E)^{*}
$$

Далее, каждое пространство связностей допускает $\mathscr{G}$-эквивариантное отображение $F: \mathscr{A}(E) \rightarrow \Omega^{2}(u(E))$, переводящее связность в ее форму кривизны.

Напомним, что для пространства $\mathscr{A}(L)$ всех $\mathrm{U}(1)$-связностей на линейном расслоении $L$ “отображение моментов” $F$ относительно групшы $\mathscr{G}$ :

$$
F: \mathscr{A}(L) \rightarrow \Omega^{2}
$$

сопоставляюшее связности $а$ ее форму кривизны $F(a)$, расслаивается на орбиты калибровки над $H^{1}(M, \mathbb{R}) / H^{1}(M, \mathbb{Z})($ см. [15]). Поэтому любая орбита связностей на $L$ определяется с точностью до "якобиана" $H^{1}(M, \mathbb{R}) / H^{1}(M, \mathbb{Z})$ как 2-форма на $M$, а именно форма кривизны этих связностей, и класс когомологий $\left[\frac{1}{2 \pi i} F(a)\right]=c_{1}(L)$. 
В общем случае действие калибровочной групшы $\mathscr{G}(E)$ на пространстве $\Omega^{2}($ End $E)$ определяет векторное расслоение $\pi: \mathscr{H}^{2} \rightarrow \mathscr{B}(E)^{*}$ со слоем $\Omega^{2}(\operatorname{ad} E)$ и отображение $F$ - его сечение.

Как мы уже знаем, конформная структура на $X$, определенная выбором римановой метрики $g$, задает $*$-разложение $\Omega^{2}(\operatorname{ad} E)=\Omega^{2+}(\operatorname{ad} E) \oplus \Omega^{2-}(\operatorname{ad} E)$ и, следовательно, разложение векторных расслоений в прямую сумму $\mathscr{H}^{2}=\mathscr{H}^{2+} \oplus \mathscr{H}^{2-}$ двух расслоений $\pi \pm: \mathscr{H}^{2 \pm} \rightarrow \mathscr{B}(E)^{*}$ со слоем $\Omega^{2 \pm}(\operatorname{ad} E)$ и разложение сечения $F$ в сумму $F=F^{+}+F^{-}$, где $F^{ \pm}-$сечения векторных расслоений $F^{ \pm}: \mathscr{B}(E) \rightarrow \mathscr{H}^{ \pm}$. Эти сечения уже имеют непустые множества нулей как подмногообразия $\mathscr{B}(E)$ :

(1) $\left(F^{+}\right)_{0}=\mathscr{M}_{\text {asd }}^{g}$,

(2) $\left(F^{-}\right)_{0}=\mathscr{M}_{\mathrm{sd}}^{g}$,

- многообразия модулей антиинстантонов и инстантонов, которые могут рассматриваться как конечномерные орбифолды виртуальных размерностей

$$
\begin{aligned}
\operatorname{vdim} \mathscr{M}_{\mathrm{asd}}^{g} & =8 c_{2}(E)-2 c_{1}^{2}(E)-3\left(b_{2}^{+}+1\right), \\
\operatorname{vdim} \mathscr{M}_{\mathrm{sd}}^{g} & =8 c_{2}(E)-2 c_{1}^{2}(E)-3\left(b_{2}^{-}+1\right) .
\end{aligned}
$$

(Здесь мы просто расширили $\mathrm{SU}(2)$-случай $§ 2$ на $\mathrm{U}(2)$-случай.)

Легко видеть, что $\mathscr{M}_{\mathrm{asd}}^{g}$ зависит от $w_{2}(E)=c_{1}(E)(\bmod 2)$ и от неположительного числа $p_{1}(E)=c_{1}(E)^{2}-4 c_{2}(E)$. Зависимость пространства модулей инстантонов от топологического типа расслоения $E$ может быть отмечена параметрами $\mathscr{M}_{\mathrm{asd}}^{g}\left(w_{2}, p_{1}\right)$ (соответственно $\left.\mathscr{M}_{\mathrm{sd}}^{g}\left(w_{2}, p_{1}\right)\right)$.

Напомним элементарные конструкции четырехмерной римановой геометрии.

В произвольной точке $p \in X$ на касательном пространстве $T_{p}=\mathbb{R}^{4}$ и его комплексификации $T^{\mathbb{C}}=\mathbb{C}^{4}$ определены изоморфизм $g^{\mathbb{C}}: T^{\mathbb{C}} \rightarrow\left(T^{\mathbb{C}}\right)^{*}$ и комплексная квадрика $Q \subset \mathbb{P}^{3}=\mathbb{P} T_{p}^{\mathbb{C}}$, заданные метрикой $g$. Как всегда, такая невырожденная комплексная квадрика есть прямое произведение

$$
Q_{p}=q_{p}^{+} \times q_{p}^{-}, \quad q_{p}^{ \pm}=\mathbb{C P}^{1}, \quad q_{p}^{ \pm}=\mathbb{P} W_{p}^{ \pm}\left(\cong \mathbb{C P}^{1}\right) .
$$

Поэтому для $p \in X$

$$
T_{p}^{\mathbb{C}}=\operatorname{Hom}\left(W_{p}^{-}, W_{p}^{+}\right)=W_{p}^{-*} \otimes W_{p}^{+} .
$$

Но квадратичная форма $g_{p}$ вешественна и положительна, следовательно, она определяет новую вещественную структуру $J: W_{p}^{ \pm} \rightarrow W_{p}^{ \pm}, J^{2}=-\mathrm{id}$, не имеющую вещественных векторов (кватернионное умножение), с точностью до одновременной гомотетии пространств $W^{ \pm}$, т.е. эрмитову метрику и совместимую симплектическую структуру на $W_{p}^{ \pm}$с точностью до гомотетии. Поэтому

$$
T_{p}=\operatorname{Hom}_{J}\left(W_{p}^{-}, W_{p}^{+}\right)
$$

- пространство гомоморфизмов, сохраняюших вешественную структуру $J$.

Далее, над точкой $p \in M$ проективное пространство $\mathbb{P}_{p}^{5}=\mathbb{P} \Lambda^{2} T_{p}^{\mathbb{C}}$ содержит две квадрики: $\Lambda^{2} g_{p}^{\mathbb{C}}$ и грассманиан $G$ прямых $\mathbb{P} T_{p}^{\mathbb{C}}$ или квадрику Клейна. Обычно $\Lambda^{2} g^{\mathbb{C}}$ рассматривается как отождествление $\Lambda^{2} T^{\mathbb{C}}=\Lambda^{2} T^{\mathbb{C} *}$. Тогда $G$ можно представлять как самосопряженный оператор

$$
*: \Lambda^{2} T^{\mathbb{C} *} \rightarrow \Lambda^{2} T^{\mathbb{C} *} ; \quad *^{2}=\mathrm{id} .
$$


Это и есть *-оператор Ходжа с собственными подпространствами

$$
\Lambda^{2} T^{\mathbb{C} *}=\Lambda_{\mathbb{C}}^{+} \oplus \Lambda_{\mathbb{C}}^{-}, \quad \text { где }\left.*\right|_{\Lambda^{ \pm}}= \pm \cdot \mathrm{id},
$$

задаюший наше разложение.

Геометрически, пересечения $G \cap \mathbb{P} \Lambda_{\mathbb{C}}^{ \pm}=q^{ \pm}$дают семейства образуюших квадрики $Q_{p}$ на грассманиане $G$.

Для однозначного определения эрмитовой структуры на $W^{ \pm}$мы должны фиксировать некоторую эрмитову структуру на $\Lambda^{2} W^{ \pm}$. Тогда эрмитова структура на $W^{ \pm}$ определяет изоморфизмы

$$
\overline{W_{p}^{ \pm}}=\operatorname{Hom}_{\mathbb{C}}\left(W_{p}^{ \pm}, \mathbb{C}\right) ; \quad \overline{W_{p}^{ \pm}} \otimes W_{p}^{ \pm}=\operatorname{End} W_{p}^{ \pm}
$$

и

$$
\operatorname{ad} W_{p}^{ \pm}=\Lambda_{\mathbb{C} p}^{ \pm} .
$$

Если $\pi_{\text {ad }}:$ End $W_{p}^{ \pm} \rightarrow \operatorname{ad} W_{p}^{ \pm}$- стандартная проекция, то мы имеем квадратичное отображение

$$
\begin{gathered}
\phi_{ \pm}: W_{p}^{ \pm} \rightarrow \Lambda_{\mathbb{C} p}^{ \pm}, \\
\phi_{ \pm}\left(\Phi_{p}\right)=\pi_{\mathrm{ad}}(\Phi \otimes \bar{\Phi}) \quad \text { для } \Phi \in W_{p}^{ \pm} .
\end{gathered}
$$

Геометрически это означает, что если $\mathbb{P} \Phi_{p} \in \mathbb{P} W^{ \pm}=q^{ \pm}$, то $\mathbb{P} \phi_{ \pm}\left(\Phi_{p}\right) \in \mathbb{P} \Lambda_{\mathbb{C} p}^{ \pm}-$ просто точка пересечения касательных к конике $q^{ \pm}$, взятых в точках $\mathbb{P} \Phi_{p}$ и $\overline{\mathbb{P} \Phi_{p}}$.

Далее, относительно вещественной структуры оператор $*$ вещественен и разложение на собственные подпространства вещественно тоже. Коники $q^{ \pm} \in \mathbb{P} \Lambda_{\mathbb{C} p}^{ \pm}$не имеют вешественных точек, следовательно, отображения $\phi_{ \pm}$вешественны и $\Phi_{p} \neq 0 \Longrightarrow$ $\phi\left(\Phi_{p}\right) \neq 0$. Отображения $\phi_{ \pm}(5.11)$ вешественны и $\operatorname{ad}_{J} W_{p}^{ \pm}=\Lambda_{p}^{ \pm}$, где $J$ - вешественная структура.

Читатель уже заметил, что в этом параграфе, в отличие от предыдущих, мы обозначаем спиноры заглавными буквами. Мы вынуждены это делать потому, что мы будем их скручивать с векторньми расслоениями. Мы можем рассмотреть спинор $\Phi_{p}$ как $g_{p}$-совместимую комплексную структуру на касательном пространстве $T_{p}=\mathbb{R}^{4}$, заданную как оператор

$$
I_{p}: T_{p}^{\mathbb{C}} \rightarrow T_{p}^{\mathbb{C}}, \quad I_{p}^{2}=-\mathrm{id},
$$

что эквивалентно разложению на собственные подпространства

$$
T_{p}^{\mathbb{C}}=T^{0,1} \oplus T^{1,0},\left.\quad I\right|_{T^{1,0}}=i \cdot \mathrm{id},\left.\quad I\right|_{T^{0,1}}=-i \cdot \mathrm{id},
$$

где

$$
T^{0,1}=\left(\mathbb{C} \cdot \Phi_{p}\right) \otimes \mathbb{C}, \quad T^{1,0}=\left(\mathbb{C} \cdot \overline{\Phi_{p}}\right) \otimes \mathbb{C} .
$$

Проективизацией этих подпространств будет пара прямых на квадрике $Q_{p} \in \mathbb{P} T_{p}^{\mathbb{C}}$, которые дают пару точек $\mathbb{P} H^{2,0}$ и $\mathbb{P} H^{0,2}$ на конике $q^{+}$в $\mathbb{P} \Lambda_{\mathbb{C} p}^{+}$, и пересечение касательных в этих точках к конике будет проективизацией симплектической формы $\omega_{p}$ (= келерова форма). И локальная компонента Ходжа $\mathbb{P} H^{1,1}=\left\langle\omega, \Lambda_{\mathbb{C} p}^{-}\right\rangle$. 
В этой интерпретации спиноров отображение $\phi$ сопоставляет комплексной структуре на $T_{p}$ симплектическую форму соответствующей эрмитовой структуры, умноженной на некоторую вещественную функцию $\psi$.

Теперь все эти объекты и конструкции могут быть глобализованы над $X$ : если $\pi_{1}(X)=0$, то проективные прямые $q_{p}^{ \pm}$становятся слоями $\mathbb{P}^{1}$-расслоения $q^{ \pm}$, а двумерные пространства $W_{p}^{ \pm}$в каждой $p \in M-$ слоями $\mathbb{C}^{2}$-расслоений $W^{ \pm}-$спинорных расслоений. Метрикой эти расслоения определены с точностью до подкрутки на линейное. Мы можем (так как $\pi_{1}=0$ ) определить подъем проективных расслоений до векторных, фиксируя первый класс Черна $c_{1}\left(W^{ \pm}\right)$при условии $c_{1}\left(W^{ \pm}\right) \equiv w_{2}(M)$ $(\bmod 2)$, где $w_{2}(M)$ - второй класс Штиффеля-Уитни многообразия $M$. Как мы уже знаем, выбор $c_{1}\left(W^{ \pm}\right)$называется выбором $\operatorname{Spin}^{\mathbb{C}}$-структуры. Комплексификация касательного расслоения представляется в виде

$$
T^{\mathbb{C}}=\operatorname{Hom}\left(W^{-}, W^{+}\right)=W^{-*} \otimes W^{+} .
$$

Метрика $g$ определяет пару SO(3)-связностей Леви-Чивита на паре расслоений $W^{ \pm}$. Добавляя к ним любую U(1)-связность $\nabla_{\text {det }}$ на линейном расслоении $L=\Lambda^{2} W^{ \pm}$, мы получим $\mathrm{U}(2)$-связности на $W^{ \pm}$. Одна из них определяет гомоморфизм $\Gamma\left(W^{+}\right) \rightarrow$ $\Gamma\left(W^{+} \otimes T_{\mathbb{C}}^{*}\right)$ пространства сечений, а композиция

$$
\Gamma\left(W^{+}\right) \rightarrow \Gamma\left(W^{+} \otimes T_{\mathbb{C}}^{*}\right) \rightarrow \Gamma\left(W^{-}\right),
$$

где последнее отображение определяется сверткой, как мы знаем, назьвается оператором Дирака (задаваемого связностью $\nabla_{\text {det }}$ на $\Lambda^{2} W^{ \pm}$).

С другой стороны, локальное отображение (5.11) может быть глобализовано до квадратичного отображения векторных расслоений:

$$
\phi_{ \pm}: W^{ \pm} \rightarrow \Lambda^{ \pm}
$$

и задает отображения сечений:

$$
\Gamma \phi_{ \pm}: \Gamma\left(W^{ \pm}\right) \rightarrow \Gamma\left(\Lambda^{ \pm}\right)
$$

Любая эрмитова связность $a \in \mathscr{A}(E)$ на $E$ и оператор Дирака (5.15) задают скрученный оператор Дирака

$$
D_{a}: \Gamma^{\infty}\left(E \otimes W^{+}\right) \rightarrow \Gamma^{\infty}\left(E \otimes W^{-}\right)
$$

по обычному правилу Лейбница. Нетрудно видеть, что скрученный оператор Дирака зависит от бесследовой аd-компоненты $a_{0}$ связности $a$ и индуцированной связности $\nabla_{\text {det }}$ на линейном расслоении $L=\operatorname{det}\left(E \otimes W^{ \pm}\right)=\Lambda^{2} E \otimes \Lambda^{2} W^{ \pm}$. Таким образом,

$$
\mathscr{A}(\operatorname{ad} E) \times \mathscr{A}(L)
$$

является пространством параметров семейства операторов. Для превращения этого семейства и всех наших пространств в банаховы пространства без ограничения общности мы можем потребовать, чтобы пространства связностей были $L_{k}^{p}$-пространствами, а калибровочные преобразования - преобразованиями класса $L_{k+1}^{p}$. 
Семейство операторов (5.18) эквивариантно по действию калибровочной групшы. Поэтому мы можем рассмотреть это семейство как гомоморфизм $D$ векторньх расслоений:

$$
\begin{aligned}
& \Gamma^{+}=\left(\Gamma\left(E \otimes W^{+}\right) \times \mathscr{A}(\operatorname{ad} E) \times \mathscr{A}(L)\right) / \mathscr{G}_{E} \rightarrow \mathscr{A}(\operatorname{ad} E) \times \mathscr{A}(L) / \mathscr{G}_{E}, \\
& \Gamma^{-}=\left(\Gamma\left(E \otimes W^{-}\right) \times \mathscr{A}(\operatorname{ad} E) \times \mathscr{A}(L)\right) / \mathscr{G}_{E} \rightarrow \mathscr{A}(\operatorname{ad} E) \times \mathscr{A}(L) / \mathscr{G}_{E}
\end{aligned}
$$

над базой

$$
\mathscr{A}(\operatorname{ad} E) \times \mathscr{A}(L) / \mathscr{G}_{E}=\mathscr{B}(\operatorname{ad} E) \times \Omega^{2} .
$$

Выберем форму $\omega \in \Omega^{2}$ и рассмотрим пространство

$$
\mathscr{A}_{\omega}(L)=\{\nabla \in \mathscr{A}(L) \mid F(\nabla)=\omega\} .
$$

Тогда

$$
\mathscr{A}(\operatorname{ad} E) \times \mathscr{A}_{\omega} / \mathscr{G}_{E}=\mathscr{B}(\operatorname{ad} E) .
$$

Рассмотрим ограничение векторного расслоения (5.20) на такое подпространство:

$$
\Gamma^{+}=\left(\Gamma\left(E \otimes W^{+}\right) \times \mathscr{A}(\operatorname{ad} E) \times \mathscr{A}_{\omega}\right) / \mathscr{G}_{E} \rightarrow \mathscr{A}(\operatorname{ad} E) / \mathscr{G}_{E}
$$

Для тройки

$$
\left(a_{0}, \nabla_{\mathrm{det}}, \phi\right) \in \mathscr{A}(\operatorname{ad} E) \times \mathscr{A}_{\omega} \times \Gamma\left(E \otimes W^{+}\right)
$$

мы имеем систему уравнений

$$
\begin{gathered}
D_{a_{0}}^{\nabla_{\operatorname{det}}}(\Phi)=0, \\
F_{a_{0}}^{+}=-(\Phi \otimes \bar{\Phi})_{00},
\end{gathered}
$$

где $-(\Phi \otimes \bar{\Phi})_{00}$ - “двойная" бесследовая компонента End $E \otimes$ End $W^{+}$.

Пространство решений этой системы по модулю действия калибровочной группы

$$
\mathscr{M}_{B}\left(g, \omega, f, p_{1}\right), \quad \text { где } f=c_{1}(E)+c_{1}\left(W^{+}\right) \in H^{2}(M, \mathbb{Z})
$$

и $p_{1}$ - число Понтрягина, называется пространством модулей В-монополей. Это пространство модулей вложено в векторное расслоение

$$
i: \mathscr{M}_{B}\left(g, \omega, f, p_{1}\right) \rightarrow \Gamma^{+}
$$

и содержит в качестве тривиальной компоненты тройки типа $\left(a_{0}, \nabla_{\text {det }}, 0\right)$, т.е. $\mathscr{M}_{\text {asd }}$, которое содержится в нулевом сечении векторного расслоения $\Gamma^{+}$.

Заметим, что уравнение В-монополя (5.25) - прямое обобшение уравнения А-монополя (4.37). Однако легко видеть, что уравнение (5.25) не имеет абелева аналога. Многообразие модулей В-монополей было использовано в [16] как пленка бордизма, связьваюшего многообразие модулей инстантонов с классами Зайберга-Виттена.

Далее, пусть

$$
\mathscr{M}_{B}\left(g, \omega, f, p_{1}\right)^{i r}=\left\{\left(a_{0}, \nabla_{\text {det }}, \Phi\right) \mid \Phi \neq 0\right\}
$$

- подмножество нетривиальных решений, а $\overline{\mathscr{M}_{B}\left(g, \omega, f, p_{1}\right)^{i r}}-$ его замыкание в $\Gamma^{+}$.

Рассмотрим пересечение

$$
\mathscr{M}_{\text {lim }}=\mathscr{M}_{\text {asd }} \cap \overline{\mathscr{M}_{B}\left(g, \omega, f, p_{1}\right)^{i r}}
$$

где $\mathscr{M}_{\text {asd }}$ мы рассматриваем как подмножество нулевого сечения $\Gamma^{+}$.

Описание $\mathscr{M}_{\text {lim }}$ просто, но не тривиально. Оно получается как следствие описания инфинитезимальной деформации решения уравнения неабелева монополя, к которому мы переходим в следующем разделе. 
5.2. Инфинитезимальная теория деформации монополя вблизи инстантона. Так же, как и для инстантонов, принципиальньй вопрос о сушествовании решений уравнения неабелева монополя может быть решен чисто теоретически при асимптотических условиях на топологические параметры. Практически же удобно воспользоваться сушествованием приводимых решений для построения решения уравнения неабелева монополя, вьпускаемого из инстантона подскока (см. [4], [5]).

Исследование инфинитезимальной деформации уравнения неабелева монополя показывает, что пределами неабелевых монополей при стремлении спинорной части решения к нулю будут инстантоны $a \in \mathscr{M}_{\text {asd }}$, допускающие нетривиальное ядро скрученного оператора Дирака: $\operatorname{ker} D_{a}>0$. В общем случае верно и обратное - из инстантона подскока можно вьпустить по меньшей мере формальное решение уравнения неабелева инстантона.

Линеаризация системы (5.25) фредгольмова:

$$
\begin{gathered}
D_{a} \delta \Phi+\delta a * \Phi=0, \\
\left.d_{a}^{+}(\delta a)=-(\delta \Phi \otimes \bar{\Phi}+\Phi \otimes \overline{\delta \Phi})\right)_{00},
\end{gathered}
$$

где $\delta a \in \Omega^{1}($ End $E), \delta \Phi \in \Gamma\left(E \otimes W^{+}\right)$- компоненты касательного вектора в точке $(a, \Phi), \mathrm{a} *$, как всегда, обозначает клиффордово умножение.

Таким образом, если мы начинаем с пары $(a, 0)$, то тогда мы получаем из первого уравнения $D_{a} \delta \Phi=0$, т.е. $a \in \mathscr{M}_{1}\left(g, \omega, f, p_{1}\right)$ - инстантон подскока.

Рассмотрим однопараметрическое дифференцируемое семейство решений системы $(5.25)\left(a_{t}, \Phi_{t}\right), t \in[0, \varepsilon]$, где

$$
D_{a_{t}} \Phi_{t}=0, \quad F_{a_{t}}^{+}=\left(\Phi_{t} \otimes \overline{\Phi_{t}}\right)_{00} .
$$

Разложение в ряд Тейлора этого семейства по параметру $t$ дает нам

$$
\Phi_{t}=\Phi_{0}+\sum_{i} \Phi_{i} t^{i}, \quad a_{t}=a+\sum_{i} \omega_{i} t^{i}
$$

где $\omega_{i} \in \Omega^{1}($ End $E)$.

Для любой формы $\omega \in \Omega^{1}($ End $E)$ мы имеем равенство

$$
D_{a+\omega} \Phi=D_{a} \Phi+\omega * \Phi .
$$

Следовательно, первое уравнение нашей системы дает следующее условие:

$$
\sum_{i} D_{a} \Phi_{i} t^{i}+\sum_{i, j} \omega_{i} * \Phi_{j} t^{i+j}=0 .
$$

Для разложения второго уравнения системы по $t$ используем формулу

$$
F_{a+\omega}^{+}=F_{a}^{+}+d_{a}^{+}(\omega)+(\omega \Lambda \omega)^{+}
$$

(см., например, $[2 ; 2.1 .14]$ ). Тогда в качестве второго уравнения получаем

$$
\sum_{i, j} \Phi_{i} \otimes \overline{\Phi_{j}} \cdot t^{i+j}=\sum_{i} d_{a}^{+}\left(\omega_{i}\right) t^{i}+\sum_{i, j}\left(\omega_{i} \Lambda \omega_{j}\right)^{+} t^{i+j} .
$$


Приравнивая выражения при одинаковых степенях в (5.33) и (5.35), мы получаем новую систему уравнений

$$
\begin{gathered}
D_{a} \Phi_{k}=-\sum_{i+j=k} \omega_{i} * \Phi_{j} \\
d_{a}^{+}\left(\omega_{k}\right)=\sum_{i+j=k}\left(\Phi_{i} \otimes \overline{\Phi_{j}}-\left(\omega_{i} \Lambda \omega_{j}\right)^{+}\right) .
\end{gathered}
$$

Очевидно, для $\Phi_{1}$ и $\omega_{1}$ имеем

$$
D_{a} \Phi_{1}=0, \quad d_{a}^{+}\left(\omega_{1}\right)=0
$$

Геометрически это означает, что

$$
\Phi_{1} \neq 0 \Rightarrow a \in \mathscr{M}_{1}\left(g, \omega, f, p_{1}\right)
$$

и $\omega_{1}-$ касательньй вектор к $\mathscr{M}_{\text {asd }}$ в точке $a$.

Вместе с системой (5.25) для троек

$$
\left(a, \nabla_{\text {det }}, \Phi\right) \in \mathscr{A}(\operatorname{ad} E) \times \mathscr{A}_{\omega} \times \Gamma\left(E \otimes W^{+}\right)
$$

рассмотрим систему уравнений

$$
\begin{gathered}
D_{a}^{\nabla_{\operatorname{det}}}(\Phi)=0 \\
F_{a}^{+}=0 .
\end{gathered}
$$

Пространство решений этой системы по модулю действия калибровочной групшы $\mathscr{M P}\left(X, p_{1}(E), c+c_{1}(E)\right)$ отображается проекцией пары (связность + спинор) на первую компоненту

$$
f: \mathscr{M} P\left(X, p_{1}(E), c+c_{1}(E)\right) \rightarrow \mathscr{M}_{\text {asd }}
$$

в пространство модулей SO(3)-инстантонов, и образом этого отображения

$$
\mathscr{M}_{1}^{g, \omega}\left(c+c_{1}(E), p_{1}(E)\right)=f\left(\mathscr{M} P\left(X, p_{1}(E), c+c_{1}(E)\right)\right) \subset \mathscr{M}_{\text {asd }}
$$

будет пространство модулей инстантонов подскока (см. [15; 1.1.26]).

Нормальный конус подмногообразия инстантонов подскока $\mathscr{M}_{1}$ может быть описан следуюшим образом:

$$
\left(C \mathscr{M}_{1}\right)_{a}=\mathscr{O}_{\mathbb{P}\left(\operatorname{ker} D_{a}\right)}(-1) \otimes \operatorname{coker} D_{a}
$$

Мы можем рассматривать первое приближение $\left(a, \Phi_{1}\right)$ как точку на $\mathscr{M}_{1}$ (в дальнейших рассмотрениях мы будем опускать лишние индексы за редким исключением, когда это может привести к недоразумению).

Для $\Phi_{2}$ мы имеем из $(5.32)$

$$
D_{a} \Phi_{2}=-\omega_{1} * \Phi_{1}
$$


Чтобы получить приближение второго порядка для спинорного поля, необходимо выбрать $\omega_{1}$ так, чтобы

$$
\operatorname{im} D_{a} \cap \operatorname{im} \Psi_{\Phi_{1}} \neq \varnothing
$$

где $\Psi_{\Phi_{1}}-$ гомоморфизм,

$$
\Psi_{\Phi_{1}}: T_{a} \mathscr{M}_{\mathrm{asd}} \rightarrow \Gamma\left(E \otimes W^{-}\right),
$$

при котором $\omega$ отображается на $\omega * \Phi$.

Мы можем интерпретировать касательное пространство $T_{a} \mathscr{M}_{\text {asd }}$ как подмножество в пространстве всех 1-фором

$$
T_{a} \mathscr{M}_{\mathrm{asd}}=\left\{\omega \in \Omega_{1}(\operatorname{ad} E) \mid d_{a}^{+}(\omega)=0, d_{a}^{*}(\omega)=0\right\} .
$$

Пусть $a$ - инстантон подскока такой, что ядро $D_{a}$ одномерно (общий случай) и $\Phi_{1} \in$ $\operatorname{ker} D_{a}$ (второй член фильтрации Брилля-Нётера вкладывается в $\mathscr{M}_{1}$ как подсхема особенностей с нормальным конусом $\left(C \mathscr{M}_{1}\right)_{a}$, описанньм в $\left.(5.42)\right)$.

Тогда естественно представить касательное пространство $T_{a} \mathscr{M}_{1}$ следуюшим обра30м:

$$
T_{a} \mathscr{M}_{1}=\left\{\omega \in T_{a} \mathscr{M}_{\text {asd }} \mid \Psi_{\Phi_{1}}(\omega) \subset \operatorname{im} D_{a}\right\} .
$$

В регулярной точке $\mathscr{M}_{1}$ нормальньй конус представляется просто как

$$
C_{a}=\operatorname{Hom}\left(\operatorname{ker} D_{a}, \operatorname{coker} D_{a}\right)
$$

и его размерность совпадает с рангом coker $D_{a}$. В этом случае виртуальная размерность подмногообразия инстантонов подскока равна

$$
v \cdot \operatorname{dim} \mathscr{M}_{1}=\operatorname{dim} \mathscr{M}_{\mathrm{asd}}-\operatorname{rk} \operatorname{ker} D_{a} \cdot \operatorname{rkcoker} D_{a}
$$

и совпадает с размерностью

$$
\operatorname{dim} T_{a} \mathscr{M}_{1}=\operatorname{dim} \mathscr{M}_{\text {asd }}-\operatorname{rkcoker} D_{a},
$$

а ранг нормального конуса

$$
\operatorname{rk} C_{a}=\operatorname{rkcoker} D_{a}
$$

(см. [15]).

Формально необходимо различать два случая: когда $\operatorname{dim} \mathscr{M}_{1}>0$ и когда $\operatorname{dim} \mathscr{M}_{\text {asd }}$ $=\operatorname{rk}$ coker $D_{a}$ (жесткий инстантон подскока). Мы рассмотрим их параллельно.

5.3. Построение формального решения. Система (5.36) очень похожа на рекурсивное задание формального решения исходной системы (5.25). Эта рекурсивная система может быть разрешена, если на каждом шаге мы сможем найти прообраз для правых частей первого и второго уравнений. Мы начнем со второго. Для доказательства существования прообразов у правых частей при дополнительном условии $d_{a}^{*}\left(\omega_{k}\right)=0$ заметим, что в “деформационном комплексе" (см. [6; 4.2.26]) условие антиавтодуальности связности $а$ означает, что это настоящий комплекс и мы можем рассматривать его когомологии $H_{a}^{0}, H_{a}^{1}, H_{a}^{2}$, причем

$$
H_{a}^{1} \cong \operatorname{ker} \delta_{a}, \quad H_{a}^{2} \cong \operatorname{coker} d_{a}^{+} \cong \operatorname{ker} d_{a}
$$


где оператор $\delta_{a}=d_{a}^{*}+d_{a}$ имеет индекс ind $\delta_{a}=s>0$ (см. [6; 4.2.27]). Первые когомологии $H_{a}^{1}$ геометрически есть просто касательное пространство форм к многообразию модулей инстантонов. В наших рассмотрениях такие формы сушествуют. Несложная редукция одного из утверждений теоремы 3.1 из [10] дает тривиальность ядра ker $d_{a}$ при условии, что связность неприводима, и это влечет coker $d_{a}^{+}=0$ (отметим, что в обшем случае это верно и для приводимых связностей). А из нетривиальности $H_{a}^{1}$ следует, что мы можем выбрать $\omega_{k}$, удовлетворяюшее условию кулоновской калибровки $d_{a}^{+}\left(\omega_{k}\right)=0$.

Очевидным образом такие формы $\omega_{k}$ определены с точностью до $T_{a} \mathscr{M}_{\text {asd }}-$ касательного пространства к многообразию модулей инстантонов.

Перейдем к рассмотрению первого уравнения системы (5.36). Как было отмечено вьше, касательное пространство к подмногообразию инстантонов подскока $T_{a} \mathscr{M}_{1} \in$ $T_{a} \mathscr{M}_{\text {asd }}$ представляется подпространством форм

$$
\left\{\omega \subset T_{a} \mathscr{M}_{\text {asd }} \mid \omega * \Phi_{1} \subset \operatorname{im} D_{a}\right\}
$$

Выберем некоторое подпространство $N_{a} \subset T_{a} \mathscr{M}_{\text {asd }}$, изоморфное слою нормального конуса $C_{a}$, так что $T_{a} \mathscr{M}_{\text {asd }}=T_{a} \mathscr{M}_{1} \oplus N_{a}$. Так же мы можем выбрать дополнение к $\operatorname{im} D_{a}$ в $\Gamma\left(E \otimes W^{+}\right)$, изоморфное coker $D_{a}$. Обозначим его $P_{a}$.

Рассмотрим композицию двух отображений:

$$
\begin{gathered}
N_{a} \rightarrow \Gamma\left(E \otimes W_{+}\right) \rightarrow P_{a}, \\
\omega \mapsto \omega * \Phi_{1} \mapsto \operatorname{pr}_{P_{a}}\left(\omega * \Phi_{1}\right) .
\end{gathered}
$$

Это линейное отображение с нулевьм ядром. В общем случае размерности $\mathrm{rk} N_{a}$ и rk coker $D_{a}$ совпадают, следовательно, это изоморфизм. Из всего сказанного следует

ПРЕДЛОЖЕНИЕ 5.1. Для любого спинора $\Phi^{-} \subset \Gamma\left(E \otimes W^{+}\right)$существует форма $\omega \in T_{a} M$ такая, что $\omega * \Phi_{1}+\Phi^{-} \subset \operatorname{im} D_{a}$.

Итак, мы можем предложить следующий индуктивный метод разрешения системы (5.36) как рекурсии. Пусть мы уже нашли $\left(\omega_{i}, \Phi_{j}\right), 1 \leqslant i \leqslant k-1,1 \leqslant j \leqslant k$. Докажем, что существует следующая пара $\left(\omega_{k}, \Phi_{k+1}\right)$. Действительно, в силу эпиморфности $d_{a}^{+}$сушествует форма $\omega_{k}^{\prime}$ такая, что

$$
d_{a}^{+}\left(\omega_{k}^{\prime}\right)=-\left(\sum_{i+j=k}\left(\omega_{i} \wedge \omega_{j}\right)^{+}+\sum_{i+j=k} \Phi_{i} \otimes \overline{\Phi_{j}}\right) .
$$

Составим следующую сумму:

$$
\Phi^{-}=-\sum_{i+j=k+1, j>1} \omega_{i} * \Phi_{j}-\omega_{k}^{\prime} * \Phi_{1}
$$

Применяя доказанное вьше предложение 5.1, получаем существование формы $\omega_{k}^{\prime \prime}$ такой, что $\Phi^{-}+\omega_{k}^{\prime \prime} * \Phi_{1} \in \operatorname{im} D_{a}$. Если положить теперь $\omega_{k}=\omega_{k}^{\prime}-\omega_{k}^{\prime \prime}$, то определится и $\Phi_{k+1}$ с точностью до ядра. Поэтому будем считать, что все $\Phi_{k}, k>1$, дают нулевую проекцию на ядро оператора Дирака, а все формы $\omega_{k}, k>1,-$ нулевую проекцию на $T_{a} \mathscr{M}_{1}$. 
Остается проверить базу индукции. Эта проверка очевидна в случае гладкого подмногообразия инстантонов подскока. Действительно, для некоторого выбранного нами спинора $\Phi_{1}$ из ядра существует форма $\omega_{1} \in T_{a} \mathscr{M}_{1}$ такая, что $\omega_{1} * \Phi_{1} \in \operatorname{im} D_{a}$, следовательно, существует и $\Phi_{2}$.

То же самое можно сделать в случае точечного инстантона подскока, т.е. когда $\operatorname{dim} \mathscr{M}_{\text {asd }}=\operatorname{rk}$ coker $D_{a}$ и пространство $T_{a} \mathscr{M}_{1}$ состоит из одной нулевой формы, только в качестве $\omega_{1}$ надо взять эту нулевую форму. Этот случай подсказьвает существование специального частного решения.

Как было указано выше, в случае точечного инстантона подскока, когда $T_{a} \mathscr{M}_{1}$ содержит только нулевую форму, мы имеем в качестве первых приближений $\Phi_{1}-$ некоторьй выбираемый нами спинор из ядра оператора Дирака $D_{a}$ (так как ядро одномерно, то этот выбор заключается просто в выборе нормы $\left.\Phi_{1}\right)$ и $\omega_{1}=0$. Если действовать дальше согласно введенной нами схеме разрешения рекурсии (5.36), мы получим интересное спешиальное формальное решение, для которого все формы с нечетными индексами $\omega_{2 k+1}=0$, все спиноры с четными $\Phi_{2 k}=0$.

Проверим сначала существование вторых и третьих приближений:

$$
D_{a}\left(\Phi_{2}\right)=\omega_{1} * \Phi_{1}=0 \Rightarrow \Phi_{2}=0 .
$$

Форма $\omega_{2}$ определяется как прообраз бесследовой компоненты

$$
d_{a}^{+}\left(\omega_{2}\right)=-\Phi_{1} \otimes \overline{\Phi_{1}},
$$

в общем случае не равной нулю, подправленньй таким образом, чтобы

$$
-\omega_{2} * \Phi_{1} \in \operatorname{im} D_{a}
$$

и тогда однозначно определен спинор $\Phi_{3}$. Для формы $\omega_{3}$ имеем

$$
d_{a}^{+}\left(\omega_{3}\right)=-\left(\omega_{1} \wedge \omega_{2}\right)^{+}-\left(\omega_{2} \wedge \omega_{1}\right)^{+}-\Phi_{1} \otimes \overline{\Phi_{2}}-\Phi_{2} \otimes \overline{\Phi_{1}}=0
$$

и поправка тоже будет нулевая, так как

$$
\Phi^{-}=-\omega_{1} * \Phi_{3}-\omega_{2} * \Phi_{2}=0 .
$$

Нетрудно видеть, что это справедливо и дальше, так как спинор с четным индексом определяется уравнением

$$
D_{a}\left(\Phi_{2 k}\right)=-\sum_{i+j=2 k} \omega_{i} * \Phi_{j}
$$

и в сумме, стояшей справа, все произведения равны нулю. Если $i$-нечетно, то $\omega_{i}=0$, если же $i$ - четно, то и $j$ - четно и, значит, $\Phi_{j}=0$. Аналогично проверяется и для $\omega_{2 k+1}$ равенство нулю.

Итак, для точечного инстантона подскока формальное решение рекуррентной задачи единственно с точностью до выбора нормы спинора $\Phi_{1}$ из ядра $\operatorname{ker} D_{a}$. Но и в том случае, когда $\operatorname{dim} \mathscr{M}_{1}>0$, мы можем решать систему (5.36), приняв за $\omega_{1}$ нулевую форму, т.е. описанное вьше формальное решение всегда содержится в пространстве формальных решений задачи (5.25). Мы будем называть это решение специальньм решением системы (5.36). 
5.4. Особенности многообразия модулей В-монополей. Для гладкости орбифолда необходима свобода действия группы на всех точках исходного факторизуемого пространства. Наоборот, особенность свидетельствует о нетривиальности стабилизатора действия группы. Для нахождения особых точек многообразия орбит по действию некоторой групшы необходимо определить те точки исходного пространства, которые определяют некоторые нетривиальные подгруппы, оставляюшие эти точки на месте - стабилизаторы.

В нашем случае описание особенностей многообразия модулей В-монополей требует работы с парами $(a, \Phi)$, но очевидно, что стабилизатор пары всегда меньше или равен стабилизатору отдельно взятой связности $a$. А для стабилизатора связности $\mathrm{St}_{\boldsymbol{a}} \subset \mathrm{U}(2)$ имеются только три возможности: $S^{1}, S^{1} \times S^{1}, \mathrm{U}(2)$, ну и, конечно, тривальная подгруппа. Последняя из трех соответствует паре, состоящей из тривиального спинора и тривиальной связности.

Случай окружности распадается на два подслучая. Если в качестве $S^{1} \subset \mathrm{U}(2)$ берется центр унитарной групшы, то эта окружность - стабилизатор точек типа $(a, 0)$, причем связность $a$ - неприводима.

Если стабилизатор $S^{1}$ пары $(a, \Phi)$ есть другая подгруппа U(2) (а именно, попадающая в максимальный тор групшы $\mathrm{PU}(2))$, то ей соответствуют приводимая связность $a=\lambda_{1} \oplus \lambda_{2}$ на некотором разложении расслоения $E=L_{1} \oplus L_{2}$ и спинор вида $\left(\Phi_{1} \oplus \Phi_{2}\right)$, причем $\Phi_{1}=0, \Phi_{2} \neq 0$ в соответствии с разложением расслоения $E$. Такие особенности - приводимые решения в случае $S^{1}$ - дают решения возмушенного уравнения абелева монополя.

И, наконец, стабилизатор $S^{1} \times S^{1}$ соответствует паре, состоящей из приводимой антиавтодуальной связности и нулевого спинора.

Многообразие модулей инстантонов некомпактно (в отличие от А-монополей), поэтому в схеме особенностей многообразия модулей В-монополей (на “хвосте") будут лежать предельные asd-связности - идеальные или bubbling-инстантоны.

Значит, пространство В-монополей представляет гладкий ориентированньй бордизм между пространством инстантонов и линками А-монополей. Мы не можем привести полностью рисунок этого кобордизма, потому что при всей общей наглядности он не отражает сложности учета всех стратов особенностей. Задача напоминает задачу учета всех стратов нестабильных расслоений как стратов Морса для функционала Янга-Миллса в двумерном случае (см. [2]) для расслоений большого ранга. Как и в двумерном случае, многие страты и их пересечения можно описать, но нам необходимо описание вкладов пересечений всех стратов, а таковое пока отсутствует. Поэтому мы не приводим ссылки на работы, содержащие промежуточные (хотя и интересные) результаты в этом направлении.

С технической точки зрения выбор соболевских норм позволяет пополнить пространство $\mathscr{B}$ и компактифицировать по Фриду-Уленбек многообразие модулей инстантонов

$$
\overline{\mathscr{M}_{\text {asd }}^{g}\left(w_{2}, p_{1}\right)}=\bigcup_{p_{1}^{\prime}>p_{1}} \mathscr{M}_{\text {asd }}^{g}\left(w_{2}, p_{1}^{\prime}\right)
$$

(см. [6]). Таким образом, определен фундаментальньй цикл

$$
\left[\overline{\mathscr{M}_{\text {asd }}^{g}\left(w_{2}(M)+w_{2}(E), p_{1}\right)}\right]
$$

многообразия модулей инстантонов. 
Подмногообразия инстантонов, представляющихся в виде пределов монополей

$$
\mathscr{M}_{\lim }(\omega, f) \subset \overline{\mathscr{M}_{\mathrm{asd}}^{g}\left(w_{2}(E)+w_{2}(M), p_{1}\right)}
$$

(см. (5.28)), определяют набор фундаментальных классов $\left[\mathscr{M}_{\lim }(\omega, f)\right]=[f]$. Та-

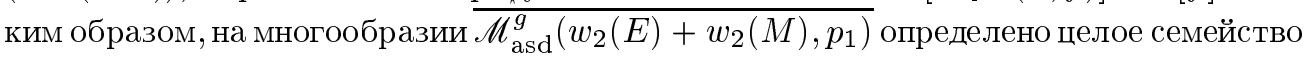
фундаментальных циклов

$$
[f], f \in H^{2}(M, \mathbb{Z}) .
$$

Заметим, что согласно нашим результатам это семейство фундаментальных классов многообразий модулей инстантонов подскока, т.е. первых элементов фильтрации Брилля-Нётера (см. [15] и [21]).

В этом случае мы можем определить фундаментальньй класс подмногообразия

$$
[f] \subset \overline{\mathscr{M}_{\text {asd }}^{g}\left(w_{2}(E)+w_{2}(M), p_{1}\right)}
$$

как класс Черна индексного расслоения оператора Дирака:

$$
[f]=c_{\text {ind } D_{a}+1}\left(\operatorname{IND} D_{a}\right), \quad \operatorname{IND} D_{a}=\operatorname{coker} D_{a}-\operatorname{ker} D_{a},
$$

где ind $D_{a}=\operatorname{rk} \operatorname{IND} D_{a}$ вычисляется по формуле Атья-Зингера.

Комбинация классов Черна всех страт и дает возможность вычислить классы Зайберга-Виттена в терминах классов Кронхеймера-Мровки и обратно. Однако подчеркнем еще раз, полное описание комбинаторики стратов и их пересечений пока что не представлено.

\section{§. Дополнение: гладкости и узлы}

Теперь уже ни для кого не секрет, что и классические инварианты Дональдсона и Зайберга-Виттена слишком слабы для успешной классификации 4-многообразий. Однако с их помошью можно различать результаты хирургических операций, сохраняющих топологическую структуру и меняющих гладкую.

Предположим, 4-многообразие $X$ содержит гладкий 2-тор $T^{2}$ с индексом самопересечения 0. Это означает, что достаточно малую трубчатую окрестность этого тора мы можем отождествить с $T^{2} \times D^{2}$. Пару

$$
T^{2} \times D^{2} \subset X
$$

назовем элементарным филагом.

Два элементарных флага $T^{2} \times D^{2} \subset X_{1}$ и $T^{2} \times D^{2} \subset X_{2}$ мы можем склеить вдоль $T^{2} \times D^{2}$, сохраняя проекцию на $D^{2}$. Результат

$$
X_{1} \#_{T^{2}} X_{2}
$$

называется расслоенной связной суммой вдоль $T^{2} \times D^{2}$. (Заметим, что эта конструкция в общем случае не однозначно определяет изотопический тип склейки. Годится любой из них.) Если $X_{i}$ были симплектические (или комплексные) многообразия, а $T^{2}$ - симплектический (комплексный) тор, то расслоенная связная сумма может быть снабжена симплектической (комплексной) структурой (конечно, не однозначно). 
Например, результат $E(1)$ раздутия девяти базисных точек пучка кривых третьей степени на $\mathbb{C P}^{2}$ обладает структурой пучка эллиптических кривых $\pi: E(1) \rightarrow \mathbb{C P}^{1}$. Выбор малой окрестности $T^{2} \times D^{2}$ любого гладкого слоя этого пучка определяет элементарньй флаг, и расслоенная связная сумма двух экземпляров такого флага дает 4-многообразие

$$
E(2)=E(1) \#_{T^{2}} E(1) .
$$

Нетрудно видеть, что $E(2)$ - подлежащее гладкое многообразие поверхности типа К3 (с выбранным эллиптическим пучком).

Итерируя эту операцию, мы получаем серию односвязных 4 -многообразий $E(n)$, $n \in \mathbb{Z}^{+},-$подлежащих гладких структур поверхностей с эллиптическим пучком с сечением и каноническим классом

$$
K_{E(n)}=(n-2)\left[T^{2}\right] \in H^{2}(E(n), \mathbb{Z}) .
$$

(Такие операции - копия получения конструктивных многообразий Калаби-Яо в [23].)

Более изящная конструкция: пусть $K \subset S^{3}$ - произвольньй узел в трехмерной сфере и $M_{K}$ - трехмерное многообразие, получающееся оснашенной хирургической операцией на $K$ из $S^{3}$. (Мы просто вырезаем трубчатую окрестность узла, применяем к ее границе - 2-тору - скручивание Дена вдоль меридиана трубки и его результат вклеиваем обратно.) Сам узел определяет окружность $S_{K}^{1} \subset M_{K}$. В 4-многообразии $S^{1} \times M_{K}$ выделен 2-тор $T_{K}^{2}=S^{1} \times S_{K}^{1}$, окрестность которого можно отождествить с $T_{K}^{2} \times D^{2}$ и получить элементарный флаг

$$
T_{K}^{2} \times D^{2} \subset S^{1} \times M_{K}
$$

Если $T^{2} \times D^{2} \subset X$ - любой другой элементарный фрлаг, то расслоенная связная сумма

$$
X_{K}=X \#_{T^{2}=T_{k}^{2}} S^{1} \times M_{K}
$$

дает нам новое 4-многообразие.

Очевидно, что 4-многообразие $S^{1} \times\left(S^{3} \backslash K\right)$ имеет те же гомологии, что и трубчатая окрестность $T^{2} \times D^{2}$ тора $T^{2}$ в $X$. С другой стороны, склейка сохраняет проекцию на $D^{2}$ и, значит,

$$
H^{2}\left(X_{K}, \mathbb{Z}\right)=H^{2}(X, \mathbb{Z}) \text { и } q_{X_{K}}=q_{X} .
$$

Предположим теперь, что

$$
\pi_{1}\left(X \backslash T^{2}\right)=1 .
$$

Тогда и многообразие $X_{K}$ односвязно и гомеоморфно $X$ (см. [8]).

Однако полином Зайберга-Виттена (4.52) различает их гладкости:

ТеОРема 6.1. Если әлементарный флаг $T^{2} \times D^{2} \subset X$ удовлетворяет условию $(6.7)$ и $b^{+}(X)>1$, mо

$$
S W_{X_{K}}=S W_{X} \cdot \Delta_{K}(t),
$$

где $t=\exp \left(2\left[T^{2}\right]\right)$, а $\Delta_{K}(t)-$ полином Александера узла $K$.

Доказательство этого замечательного равенства использует скейн-соотношения для полиномов Александера (см. [8]) и теорию Зайберга-Виттена для 3-многообразий. (В этом случае уравнение то же, только спинорное расслоение одно.) Было 
замечено (Менг, Таубс, Тураев), что SW-инварианты в трехмерном случае напрямую связаны с кручением Милнора.

Теперь в качестве стартового многообразия удобно взять поверхность $\mathrm{K} 3=E(2)$ с единицей в качестве полинома Зайберга-Виттена (см. (4.52)). Тогда для любого узла $K$

$$
S W_{E(2)_{K}}=\Delta_{K}(t), \quad t=\exp \left(2 T^{2}\right)
$$

где $T^{2}$ - слой эллиптического пучка.

Если $K$-расслоенньй узел, то многообразие $S^{1} \times M_{K}-$ расслоение над 2 -тором и

1) несет симплектическую структуру;

2) тор $T_{K}^{2}$ - симплектическое подмногообразие и

3) полином $\Delta_{K}(t)$ унимодулярный.

Если же $\Delta_{K}(t)$ не унимодулярный, то $X_{K}$ гомеоморфно К3-поверхности, но не несет никакой симплектической структуры. (Выбирая разные узлы, мы получаем бесконечное число таких многообразий.)

Используя узлы с одинаковьми полиномами Александера, но разного рода, можно попытаться построить односвязные 4-многообразия, гомеоморфные К3-поверхности, с одинаковыми SW-инвариантами, как это удалось сделать для неодносвязных 4-многообразий. Живет правдоподобная гипотеза:

$$
E(2)_{K} \text { и } E(2)_{K^{\prime}} \text { диффеоморфны }=K \text { и } K^{\prime} \text { эквивалентны. }
$$

Используя более сложные перестройки (например, линки вместо узлов или узлы большого рода), можно расширить число экзотических структур и улучшить их качество (например, Финташель и Стерн построили не симплектическую, экзотическую К3-поверхность только с одним фундаментальным классом).

Приведенные результаты и конструкции, казалось бы, приводят нас к вьводу, что инварианты, доставляемые калибровочными теориями, такие, как популярные ныне $K M_{X}$ и $S W_{X}$, слишком слабы для гладкой классификации 4-многообразий. Чтобы оспорить такой пессимистический взгляд, проведем историческую параллель: когда Ликориш построил две гомологичесие 3-сферы с одинаковыми когомологиями Флоера, Майкл Атья заметил, что результат касается только $\mathrm{SU}(2)$-когомологий. Имеются еще $\mathrm{SU}(3), \mathrm{SU}(4)$ и т. д. Мы не поленились описать в нашем обзоре неабелевы монополи (см. §5), открьваюшие бесконечную серию "неклассических" инвариантов, доставляемых все теми же калибровочньми теориями. Дело - за технической разработкой этих более сложных конструкций.

\section{СПИСОК ЛИТЕРАТУРЫ}

[1] J. Ambjørn. Quantization of Geometry, Lect. given at Les Houches, Nato Adv. Sci. Inst. Fluctuating Geometries in Statistical Mechanics and Field Theory, Session LXII, 1994.

[2] M.F. Atiyah, R. Bott. The Yang-Mills equations over Riemann surfaces // Philos. Trans. Roy. Soc. London Ser. A. 1982. V. 308. № 1505. P. 523-615.

[3] П. А. М. Дирак. Принципы квантовой механики. М.: Мир, 1960.

[4] S. Donaldson. Symplectic submanifolds and almost-complex geometry // J. Differential Geom. 1996. V. 44. P. 666-705.

[5] S. Donaldson. Lefschetz fibrations in symplectic geometry // Doc. Math. J. DMV. Extra Volume ICM Berlin. 1998. V. II. P. 309-314. 
[6] S. Donaldson, P. Kronheimer. The Geometry of Four-Manifolds. Oxford: Clarendon Press, 1990.

[7] R. Donagi, E. Witten. Supersymmetric Yang-Mills theory and integrable systems // Nuclear Phys. B. 1996. V. 460. № 2. P. 299-334; hep-th/9510101.

[8] R. Fintushel, R. Stern. Knots, links, and 4-manifolds // Invent. Math. 1998. V. 134. № 2. P. 363-400.

[9] R. Fintushel, R. Stern. Constructions of smooth 4-manifolds // Doc. Math. J. DMV. Extra Volume ICM Berlin. 1998. V. II. P. 1-10; math.GT/9907178.

[10] Д. Фрид, К. Уленбек. Инстантоны и четырехмерные многообразия. М.: Мир, 1988.

[11] M. H. Freedman, F. Luo. Selected Applications of Geometry to Low-Dimensional Topology. Marker lectures in the mathematical sciences held at the Pennsylvania State University, 1987. Providence, RI: Amer. Math. Soc., 1989.

[12] Б. Карпов. S-двойственность и исключительные расслоения // Изв. РАН. Сер. матем. 1999. T. 63. № 1. C. 107-122.

[13] P.W. Kronheimer, T.S. Mrowka. The genus of embedded surfaces in the projective plane // Math. Res. Lett. 1994. V. 1. P. 797-808.

[14] S. Mukai. On moduli spaces of bundles on K3 surfaces. II // Vector Bundles on Algebraic Varieties. Bombay colloquium. Oxford: Oxford Univ. Press, 1984. P. 341-414.

[15] В. Пидстригач, А. Тюрин. Инварианты гладкой структуры алгебраической поверхности, задаваемые оператором Дирака // Изв. РАН. Сер. матем. 1992. Т. 56 . № 2. C. $279-371$.

[16] V. Pidstrigach, A. Tyurin. Localisation of the Donaldson's Invariants along SeibergWitten Classes // Preprint. Bielefeld: Bielefeld University, 1995.

[17] D. Salamon. Spin geometry and Seiberg-Witten invariants // Preprint. Warwick: University of Warwick, 1995.

[18] N. Seiberg, E. Witten. Electric-magnetic duality, monopole condensation and confinement in $N=2$ supersymmetric Yang-Mills theory // Nuclear Phys. B. 1994. V. 426. № 1 . P. 19-52; hep-th/9407087; Corrigendum ibid. V. 430. № 2. P. 485-486.

[19] C. H. Taubes. The Seiberg-Witten invariants and symplectic forms // Math. Res. Lett. 1994. V. 1. №6. P. 809-822.

[20] C. H. Taubes. More constraints on symplectic forms from Seiberg-Witten invariants // Math. Res. Lett. 1995. V. 2. №1. P. 9-13.

[21] А. Н. Тюрин. Спин-полиномиалњные инварианты гладких структур на алгебраических поверхностях // Изв. РАН. Сер. матем. 1993. Т. 57. № 2. С. 125-164.

[22] А. Н. Тюрин. Алгебро-геометрические аспекты гладкости. 1. Полиномы Доналшдсона // УМH. 1989. Т. 44. № 3. С. 93-143.

[23] А. Н. Тюрин. Специалнная лагранжева геометрия как малая деформация алгебраической геометрии (GQP и зеркальная симметрия) // Изв. РАН. Сер. матем. 2000. Т. 64. № 2. C. $141-224$

[24] Н. А. Тюрин. Необходимое и достаточное условия деформации В-монополя в инстантон // Изв. РАН. Сер. матем. 1996. Т. 60. №1. С. 211-224.

[25] C. Vafa, E. Witten. A strong coupling test of $S$-duality // Nuclear Phys. B. 1994. V. 431. № 1-2. P. 3-77; hep-th/9408169.

[26] E. Witten. Monopoles and four-manifolds // Math. Res. Lett. 1994. V. 1. P. 769-796.

[27] E. Witten. Duality in supersymmetric gauge theories. Notes from E. Witten's lectures, Santa Cruz, 1995. 